\title{
Frequency and properties of bars in cluster and field galaxies at intermediate redshifts ${ }^{\star}$
}

\author{
F. D. Barazza ${ }^{1}$, P. Jablonka ${ }^{1,2,3}$, V. Desai ${ }^{4}$, S. Jogee ${ }^{5}$, A. Aragón-Salamanca ${ }^{6}$, G. De Lucia $^{7}$, R. P. Saglia ${ }^{8}$, C. Halliday ${ }^{9}$, \\ B. M. Poggianti ${ }^{10}$, J. J. Dalcanton ${ }^{11}$, G. Rudnick ${ }^{12}$, B. Milvang-Jensen ${ }^{13,14}$, S. Noll ${ }^{8,15}$, L. Simard ${ }^{16}$, D. I. Clowe ${ }^{17}$, \\ R. Pelló ${ }^{18}$, S. D. M. White ${ }^{7}$, and D. Zaritsky ${ }^{19}$
}

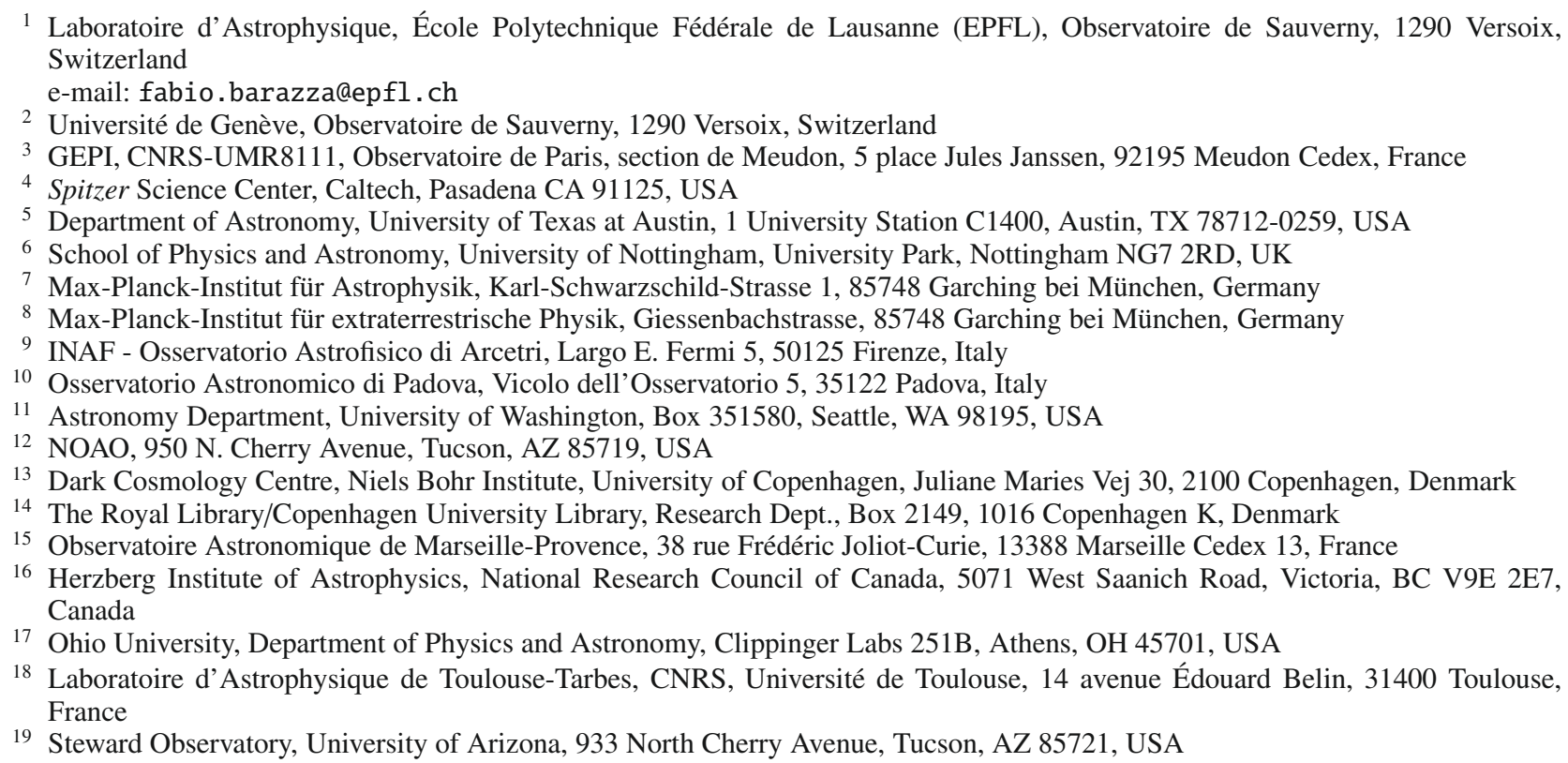

Received 9 June 2008 / Accepted 29 January 2009

\section{ABSTRACT}

\begin{abstract}
We present a study of large-scale bars in field and cluster environments out to redshifts of $\sim 0.8$ using a final sample of 945 moderately inclined disk galaxies drawn from the EDisCS project. We characterize bars and their host galaxies and look for relations between the presence of a bar and the properties of the underlying disk. We investigate whether the fraction and properties of bars in clusters are different from their counterparts in the field. The properties of bars and disks are determined by ellipse fits to the surface brightness distribution of the galaxies using HST/ACS images in the $F 814 \mathrm{~W}$ filter. The bar identification is based on quantitative criteria after highly inclined $\left(>60^{\circ}\right)$ systems have been excluded. The total optical bar fraction in the redshift range $z=0.4-0.8$ (median $z=0.60$ ), averaged over the entire sample, is $25 \%$ (20\% for strong bars). For the cluster and field subsamples, we measure bar fractions of $24 \%$ and $29 \%$, respectively. We find that bars in clusters are on average longer than in the field and preferentially found close to the cluster center, where the bar fraction is somewhat higher $(\sim 31 \%)$ than at larger distances $(\sim 18 \%)$. These findings however rely on a relatively small subsample and might be affected by small number statistics. In agreement with local studies, we find that disk-dominated galaxies have a higher optical bar fraction $(\sim 45 \%)$ than bulge-dominated galaxies $(\sim 15 \%)$. This result is based on Hubble types and effective radii and does not change with redshift. The latter finding implies that bar formation or dissolution is strongly connected to the emergence of the morphological structure of a disk and is typically accompanied by a transition in the Hubble type. The question whether internal or external factors are more important for bar formation and evolution cannot be answered definitely. On the one hand, the bar fraction and properties of cluster and field samples of disk galaxies are quite similar, indicating that internal processes are crucial for bar formation. On the other hand, we find evidence that cluster centers are favorable locations for bars, which suggests that the internal processes responsible for bar growth are supported by the typical interactions taking place in such environments.
\end{abstract}

Key words. galaxies: spiral - galaxies: structure - galaxies: clusters: general - galaxies: evolution - galaxies: formation

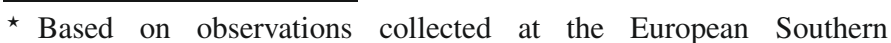
Observatory, Chile, as part of large programme 166.A-0162 (the ESO Distant Cluster Survey). Also based on observations made with the NASA/ESA Hubble S pace Telescope, obtained at the Space Telescope Science Institute, which is operated by the Association of Universities for Research in Astronomy, Inc., under NASA contract NAS 5-26555.

\section{Introduction}

There is evidence that the dynamical and secular evolution of disk galaxies is intimately connected with the presence of

These observations are associated with proposal 9476. Support for this porposal was provided by NASA through a grant from Space Telescope Science Institute. 
stellar bars. Theory and $n$-body simulations predict that bars transfer angular momentum to the outer disk, which causes the stellar orbits in the bar to become elongated and the bar amplitude to increase (Lynden-Bell 1979; Pfenniger \& Friedli 1991; Sellwood \& Wilkinson 1993; Athanassoula 2003). The growing bar becomes more and more efficient in driving gas inside the corotation radius towards the center of the disk, which can trigger starbursts (Hunt \& Malkan 1999; Regan et al. 1999; Sakamoto et al. 1999; Regan \& Teuben 2004; Bournaud \& Combes 2002; Schinnerer et al. 2002; Jogee et al. 2005; Sheth et al. 2005) and contribute to the formation of disky bulges (Kormendy 1993; Sakamoto et al. 1999; Kormendy \& Kennicutt 2004; Athanassoula et al. 2005; Jogee et al. 2005; Sheth et al. 2005; Debattista et al. 2006). The redistribution of angular momentum driven by bars is not restricted to the baryonic component, but also applies to dark matter (Weinberg 1985; Combes et al. 1990; Debattista \& Sellwood 2000; Berentzen et al. 2006). Another indication of secular evolution induced by the orbital structure and resonances in a bar potential is provided by box- or peanut-shaped bulges in inclined galaxies (Combes et al. 1990; Pfenniger \& Norman 1990; Kuijken \& Merrifield 1995; Bureau \& Freeman 1999; Martinez-Valpuesta et al. 2006; Debattista et al. 2006). These processes affect the properties of disk galaxies and therefore shape the diversity of morphologies.

While it is still unknown why a specific disk galaxy hosts a bar and an apparently similar galaxy is unbarred, it is clear that a significant fraction of bright disk galaxies appears barred in optical observations (Eskridge et al. 2000; Marinova \& Jogee 2007; Reese et al. 2007; Barazza et al. 2008). In studies carried out in the near-infrared (NIR) or in both, NIR and optical, the NIR bar fractions are typically higher (Knapen 1999; Eskridge et al. 2000; Laurikainen et al. 2004; Menéndez-Delmestre et al. 2007; Marinova \& Jogee 2007). These findings indicate that bar detection is affected by dust extinction and that studies completed at different wavelengths cannot be readily compared. This caveat is important particularly when results from local bar studies are compared with the findings of investigations at intermediate redshifts, where issues such as band shifting, surface brightness dimming, and reduced resolution further decrease the bar detection rate. In earlier studies, it was found that the bar fraction undergoes a significant intrinsic decline out to $z \sim 1$ (Abraham et al. 1999; van den Bergh et al. 2000), which was confirmed by Sheth et al. (2008). Other studies report that the bar fraction is fairly constant out to $z \sim 1$ for strong bars (Jogee et al. 2004, bar ellipticity $>0.4$ ) or all bars (Elmegreen et al. 2004; Zheng et al. 2005, see also Sect. 7), once the aforementioned effects are taken into account.

To identify and characterize bars, different methods have been applied. The most straightforward approach is to inspect images visually and assign a bar class (e.g., weak/strong bars) to each galaxy (de Vaucouleurs et al. 1991; Eskridge et al. 2000). By adopting this approach, strong bars were detected twice as frequently in near-infrared data than optical data (Eskridge et al. 2000) and the bar fraction was found to increase between Sc galaxies and later types (Odewahn 1996). Apart from visual classification, a quantitative characterization of bars has been attempted using for instance, the gravitational torque method (Block et al. 2002; Laurikainen et al. 2002; Buta et al. 2005), Fourier dissection (Buta et al. 2006; Laurikainen et al. 2006), and ellipse fits to the galaxy isophotes (Regan \& Elmegreen 1997; Abraham et al. 1999; Sheth et al. 2000, 2002, 2008; Knapen et al. 2000; Erwin \& Sparke 2002; Erwin 2005; Jogee et al. 2002a,b, 2004; Whyte et al. 2002; Elmegreen et al. 2004; Reese et al. 2007; Menéndez-Delmestre et al. 2007; Marinova \& Jogee 2007;
Barazza et al. 2008). These methods provide measurements of the bar length and shape and enable the impact of the bar on the disk to be assessed.

The vast majority of these bar studies have concentrated on field galaxy samples and estimated the bar fraction among disk galaxies and the general properties of bars and their host galaxies. First attempts have been made to relate the presence of a bar to its host galaxy properties, such as disk structure, central surface brightness, or color. On the other hand, there have been few studies of the relation between the occurrence of bars and environment. Kumai et al. (1986) studied the relative fractions of different disk galaxy types as a function of environment. They detected no increase in bar fraction inside galaxy clusters or groups, but measured a significantly higher fraction of barred disks in binary systems. This was confirmed by Elmegreen et al. (1990) and Giuricin et al. (1993), who both found that galaxies in binary systems are preferentially early type and barred. A similar result was reported by Varela et al. (2004). Interestingly, while the fraction of barred disks in clusters or groups is not higher than in the field (Kumai et al. 1986; van den Bergh 2002, 2007; Marinova et al. 2009), Thompson (1981) and Andersen (1996) presented evidence that barred galaxies in the Coma and Virgo clusters are more concentrated toward the cluster centers than unbarred disks.

We present the first study of bars in clusters at intermediate redshifts, which enables the properties of bars and their host galaxies to be studied in dense environments. We use a final sample of 945 moderately inclined disk galaxies drawn from a parent sample of 1906 disk galaxies from the ESO distant cluster survey (EDisCS, White et al. 2005). We use the available $I$-band HST/ACS images to identify and characterize bars, based on quantitative criteria. We use this sample to look for relations between the occurrence and the properties of bars and their host galaxies. For a subsample of 241 objects, for which spectroscopic redshifts and reliable cluster membership determinations are available, we look for relations between barred and unbarred galaxies and their environment. We also study a wide range of redshifts, which allows us to search for trends with increasing look-back time. The outline of the paper is as follows: in Sect. 2 we present the available data and our sample selection. The method to identify and characterize bars as well as limits of the detectability of bars in our data is described in Sect. 3. Our results in terms of bar fractions and relations between bars and host galaxy properties are presented in Sect. 4. In Sect. 5 we discuss the properties of the bars in our sample galaxies, and in Sect. 6 look at the specific distribution of barred galaxies within the clusters. The implications of our results are discussed in Sect. 7 and the summary and conclusions are given in Sect. 8. Throughout the paper, we assume a flat cosmology with $\Omega_{\mathrm{M}}=1-\Omega_{\Lambda}=0.3$ and $H_{0}=70 \mathrm{~km} \mathrm{~s}^{-1} \mathrm{Mpc}^{-1}$. Magnitudes are given in the Vega system.

\section{Sample selection from EDisCS}

The ESO Distant Cluster Survey is a study of 26 optically selected and spectroscopically confirmed galaxy systems, from rich groups to massive clusters, smoothly distributed between redshifts 0.39 and 0.96 (Halliday et al. 2004; Milvang-Jensen et al. 2008). For all systems, we have assembled three-band optical VLT deep photometry, deep NTT/SOFI near-infrared imaging, and optical VLT/FORS2 spectroscopy (White et al. 2005). We also acquired HST/ACS images in the $F 814 \mathrm{~W}$ filter for 10 fields containing the most distant clusters studied by EDisCS. The exposure time of these observations per pixel is $2040 \mathrm{~s}$, 
Table 1. Basic properties of clusters and the secondary structures.

\begin{tabular}{lccrrrrr}
\hline $\begin{array}{c}\text { Cluster/Group } \\
\text { name }\end{array}$ & $\begin{array}{c}\text { RA (J2000.0) } \\
\text { (hh mm ss) }\end{array}$ & $\begin{array}{c}\text { Dec (J2000.0) } \\
\text { (dd mm ss) }\end{array}$ & $z$ & $\begin{array}{c}\sigma \\
\left(\mathrm{km} \mathrm{s}^{-1}\right)\end{array}$ & $\begin{array}{c}R_{200} \\
(\mathrm{Mpc})\end{array}$ & $N_{\text {tot }}^{C}$ & $N_{\text {tot }}^{F}$ \\
\hline cl1037.9-1243 & 103751.4 & -124326.6 & 0.58 & 319 & 0.57 & 168 & 74 \\
cl1037.9-1243a & 103752.3 & -124449.0 & 0.43 & 537 & 1.06 & 33 & $(\ldots)$ \\
cl1040.7-1155 & 104040.3 & -115604.2 & 0.70 & 418 & 0.70 & 68 & 86 \\
cl1054.4-1146 & 105443.5 & -114619.4 & 0.70 & 589 & 0.99 & 138 & 74 \\
cl1054.7-1245 & 105443.5 & -124551.9 & 0.75 & 504 & 0.82 & 94 & 89 \\
cl1103.7-1245 & 110343.4 & -124534.1 & 0.96 & 534 & 0.77 & 0 & 76 \\
cl1103.7-1245a & 110334.9 & -124646.2 & 0.63 & 336 & 0.59 & 3 & $(\ldots)$ \\
cl1103.7-1245b & 110336.5 & -124422.3 & 0.70 & 252 & 0.42 & 6 & $(\ldots)$ \\
cl1138.2-1133 & 113810.2 & -113337.9 & 0.48 & 732 & 1.40 & 103 & 72 \\
cl1138.2-1133a & 113808.6 & -113654.9 & 0.45 & 542 & 1.05 & 7 & $(\ldots)$ \\
cl1216.8-1201 & 121645.3 & -120117.6 & 0.79 & 1018 & 1.61 & 117 & 127 \\
cl1227.9-1138 & 122753.9 & -113817.3 & 0.64 & 574 & 1.00 & 128 & 76 \\
cl1227.9-1138a & 122752.1 & -113958.7 & 0.58 & 341 & 0.61 & 8 & $(\ldots)$ \\
cl1232.5-1250 & 123230.3 & -125036.4 & 0.54 & 1080 & 1.99 & 143 & 66 \\
cl1354.2-1230 & 135409.8 & -123101.5 & 0.76 & 648 & 1.05 & 60 & 81 \\
cl1354.2-1230a & 135411.4 & -123045.2 & 0.60 & 433 & 0.77 & 7 & $(\ldots)$ \\
\hline
\end{tabular}

Notes: when more than one cluster or group is found in the EDisCS fields, they are identified with "a" or "b" appended to the name of the main cluster (structures with $\sigma<400 \mathrm{~km} \mathrm{~s}^{-1}$ are regarded as groups). The coordinates of the clusters/groups correspond to the positions of the brightest cluster/group galaxies. The redshifts $(z)$ and line-of-sight cluster/group velocity dispersions $(\sigma)$ are taken from Halliday et al. (2004) and Milvang-Jensen et al. (2008). The virial radius $\left(R_{200}\right)$ has been determined using Eq. (8) from Finn et al. (2005). $N_{\text {tot }}^{C}$ gives the total number of disk galaxies (i.e. S0-Sm/Im) in the cluster and $N_{\text {tot }}^{F}$ gives the number of disk galaxies in the corresponding field. For the structures cl1227.9-1138 and cl1227.9-1138a no Sérsic fits could be performed, because the data only became available much later and could not be included in the general analysis anymore.

except for the central $3.5^{\prime} \times 3.5^{\prime}$, which has an exposure time per pixel of $10200 \mathrm{~s}$. We completed both a visual classification of galaxy morphologies (see Sect. 2.1) and a quantitative analysis of their structural parameters (Desai et al. 2007; Simard et al. 2009). The visual classification was completed for all galaxies brighter than $I_{\text {auto }}=23 \mathrm{mag}$, where $I_{\text {auto }}$ is the SExtractor AUTO magnitude measured on the $I$-band VLT images. In this work, we adopt the same magnitude cut at $I_{\text {auto }}=23$ mag and consider only galaxies with Hubble types $\mathrm{S} 0-\mathrm{Sm} / \mathrm{Im}$, i.e. disk galaxies and lenticulars, which can also be barred ${ }^{1}$. For $\sim 90 \%$ of the sample, a Sérsic fit to the surface brightness distribution (with GIM2D, Simard et al. 2002, 2009) has been performed on the $I$-band HST/ACS images, providing the effective radius used in our analysis (see Sect. 4.3).

We select all galaxies meeting these criteria in ten fields regardless of whether they are cluster members or group/field galaxies. These fields encompass a total of 12 clusters and 4 groups. Structures with $\sigma<400 \mathrm{~km} \mathrm{~s}^{-1}$ are regarded as groups. Galaxies are considered to be cluster/group members if the integrated photometric redshift probability to be within $z \pm 0.1$ of the cluster redshift is greater than a specific limit. These limits are based on our spectroscopy and range from 0.1 to 0.35 depending on the filter set available for each particular field (White et al. 2005; Pelló et al. 2008). Cluster/group membership for objects with spectroscopic observations is defined as being within $\pm 3 \sigma_{\mathrm{cl}}$ of $z_{\mathrm{cl}}$ (Halliday et al. 2004; Milvang-Jensen et al. 2008). Photometric redshifts were determined using the methods described in Rudnick et al. (2001) and Pelló et al. (2008), and were based on the optical+near-infrared photometry. The accuracy of photometric redshifts is typically $\Delta z /(1+z) \approx 0.05 \pm 0.01$ (Pelló et al. 2008). The rest-frame magnitudes and colors were computed using the method described in Rudnick et al. (2003).

\footnotetext{
${ }^{1}$ In the remainder of the paper, we use the terms disk-galaxy sample or disk galaxies for brevity including S0s.
}

We restrict the cluster and field samples to the redshift range $z=0.4-0.8$ in order to remain in the rest-frame optical. The median photometric redshift of the total sample is 0.60 . The basic properties of the main and secondary structures and the number of objects found in these structures are given in Table 1. We show the distributions in redshift and absolute $V$ magnitude in Figs. 1 and 2, respectively. These plots include the parent sample of 1906 galaxies, to which our bar classification method (see Sect. 3) was applied. Spectroscopic redshifts are available for a subsample of 459 galaxies. For the low redshift cluster fields $(z<0.6)$ spectroscopic observations were restricted to objects with $I<22$ mag and for the high redshift cluster fields $(z>0.6)$ to $I<23$ mag (Halliday et al. 2004; Milvang-Jensen et al. 2008). This ensures that the distribution in absolute magnitude for galaxies with spectroscopic redshifts remains roughly the same over the entire redshift range $(z=0.4-0.8)$. The distributions in Figs. 1 and 2 for the cluster and field subsamples are very similar. The arrows in Fig. 2 indicate the absolute $V$-band magnitude for a Sa galaxy at $z=0.8$ corresponding to $I_{\text {auto }}=23 \mathrm{mag}$ ( $M_{V} \approx-19.6 \mathrm{mag}$, taking into account a $K$-correction). The same calculation for a Sc galaxy would result in a slightly fainter absolute $V$-band magnitude. In the following, we present our main results for both the total sample and a sample restricted to $M_{V} \leq-20 \mathrm{mag}$, which is our completeness limit (the complete sample). This shows that the incompleteness for galaxies with $M_{V}>-20$ is not biasing our results. All numbers and fractions always refer to the total sample. In Sect. 4, results based on the separation between cluster/group and field galaxies are always based on our spectroscopic data. This reduces the sample size considerably, but ensures a reliable cluster or field allocation. We estimate that a photometrically based cluster sample would have a field galaxy contamination of up to $40 \%$. Finally, we emphasize that the number of objects in the four groups is rather small and that only three bars are found in group galaxies (see also Table 1). For the remainder of the paper, we therefore 


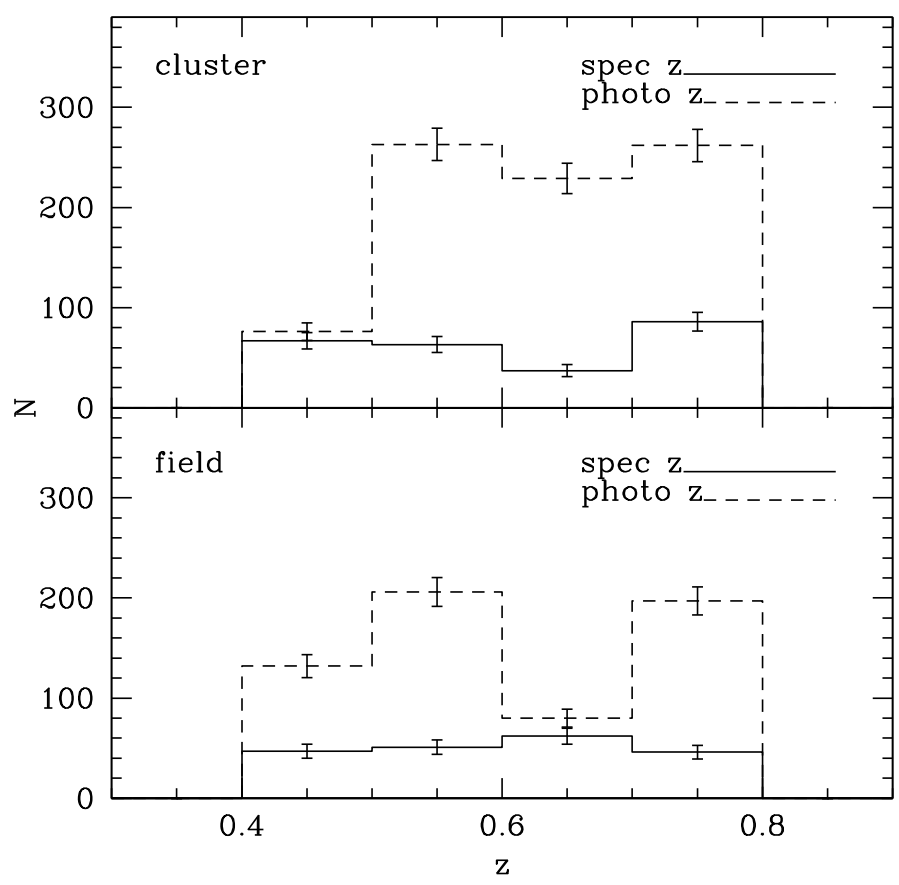

Fig. 1. Redshift distribution of the basic sample of 1906 disk galaxies. The solid line shows the distribution based on spectroscopic redshifts and the dashed line that based on photometric redshifts.

refer to the subsample of cluster/group galaxies as the cluster subsample.

\subsection{The visual classification of the galaxies}

We visually classified all galaxies brighter than $I_{\text {auto }}=23$ mag, where $I_{\text {auto }}$ is the SExtactor (Bertin \& Arnouts 1996) magnitude measured on the $I$-band VLT images and is an estimate of the total magnitude of a galaxy in the Vega system. This classification has been described in detail in Desai et al. (2007). We recall here its main features in particular those related to our purpose, i.e., the spiral galaxies. Each of the five classifiers (AAS, JJD, VD, PJ and BP) was trained on the HST WFPC2 images and visual morphological catalogs of the $0.3<z<0.5$ MORPHS clusters, using the same procedure as described in Smail et al. (1997).

Since, in this analysis, we consider possible trends of bar fraction with the Hubble type of the galaxies, we now investigate our ability to distinguish between adjacent types, e.g., Sa from Sb, Sc from Sd, etc. Galaxies in the cluster C11216-1201 $(z=0.79)$ were classified by all five classifiers and the data set for this cluster can be used to complete the most reliable statistical analysis. We found that the mean dispersion between classifiers and for all spiral galaxies was 1.2 T-type. The fraction of galaxies with a dispersion of less than $2 \mathrm{~T}$-types among galaxies was $66 \%$, and increased to $89 \%$ for 3-types ( $\mathrm{Sa}$ to $\mathrm{Sb}$ for example). These global numbers did not change if one considers early- or late-type spirals. Type $6(\mathrm{Sd})$ galaxies were identified within 3 T-types at $88 \%$, while type 1 (Sa) were at $91 \%$. The rest of the clusters were analyzed by two classifiers. Their statistics is either identical to the one of Cl1216-1201 or have slightly lower success rates. However, the ability to agree to less than 3 T-type is never lower than $60 \%$. This shows that our classification is robust and trends along the Hubble sequence can be reliably detected. In Fig. 3 we show examples of S0-Sc galaxies for cluster CL1232-1250 at $z=0.54$ and cluster CL1216-1201

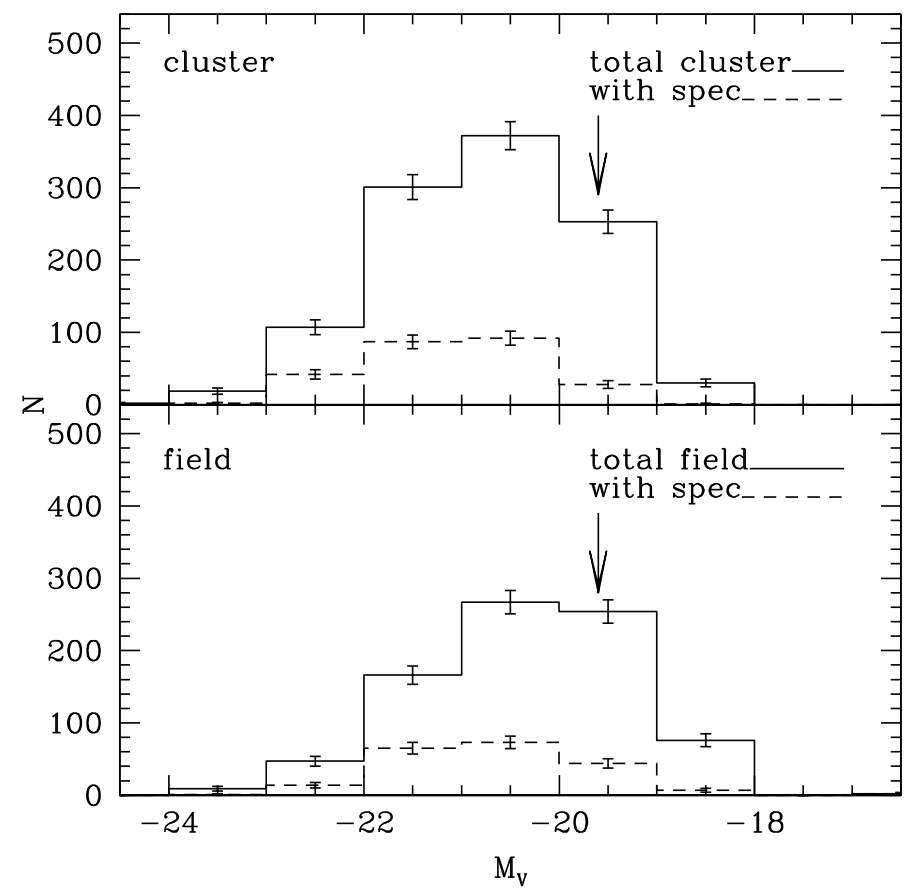

Fig. 2. Distribution of absolute $V$ magnitude for the cluster and field samples. The solid histograms show the distribution for the total sample of 1906 galaxies, whereas the dashed histograms represent the spectroscopic subsample. The arrows indicate the magnitude cut (at $M_{V} \approx$ $-19.6 \mathrm{mag}$ ) for a Sa galaxy at $z=0.8$ corresponding to $I_{\text {auto }}=23$ mag.

at $z=0.79$. The image depth and resolution are sufficient to separate Hubble types even at $z=0.79$.

\section{Bar characterization and detectability}

We first describe our method to detect and characterize bars using the $I$-band HST/ACS images and then discuss the limitations of the detectability of bars imposed by the observations used in our study.

\subsection{The detection and characterization of bars}

Our method of finding bars relies on the fact that the isophotes of bars in moderately inclined disk galaxies (i.e., with disk inclination $i<60^{\circ}$ ) have much higher ellipticities than the isophotes of the underlying disk. The ellipticities of the isophotes are derived by fitting ellipses to the surface brightness distribution of the disks. The corresponding profiles of ellipticity $(\epsilon)$ and position angle (PA) are investigated based on quantitative criteria. The method of ellipse fits has been used widely by observational studies of bars in disk galaxies (Friedli et al. 1996; Regan \& Elmegreen 1997; Abraham et al. 1999; Jogee et al. 1999; Knapen et al. 2000; Sheth et al. 2000; Laine et al. 2002; Whyte et al. 2002; Jogee et al. 2002a,b; Sheth et al. 2003; Elmegreen et al. 2004; Reese et al. 2007; Menéndez-Delmestre et al. 2007; Sheth et al. 2008). There is also strong theoretical evidence supporting this approach (Athanassoula 1992; Shen \& Sellwood 2004).

The specific method we use in this study has already been applied in earlier investigations and a detailed description can be found in the corresponding papers (Jogee et al. 2004; Marinova \& Jogee 2007; Barazza et al. 2008). We start by fitting ellipses to the images using the standard IRAF task "ellipse" via an iterative wrapper developed by Jogee et al. (2004), which (a) refines the 

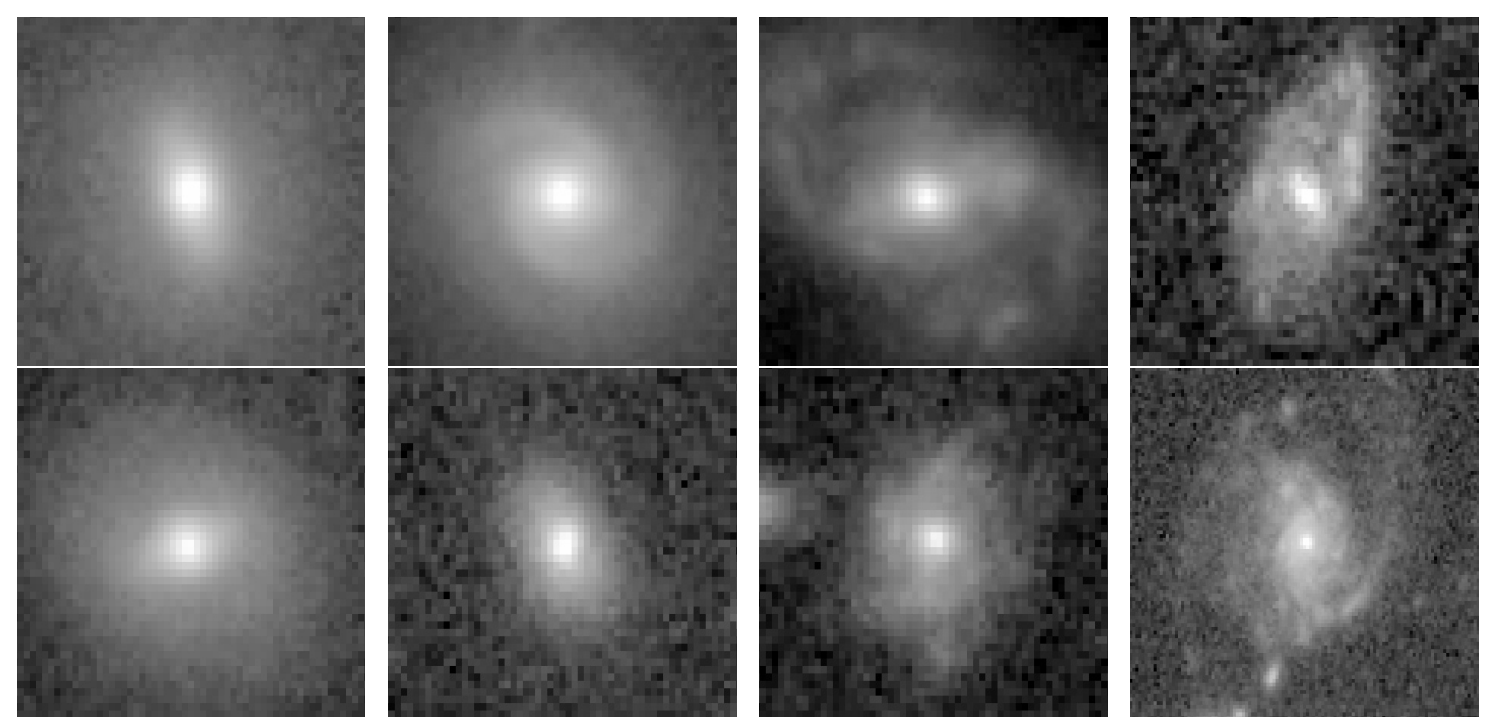

Fig. 3. From left to right examples of S0, Sa, Sb, and Sc galaxies for cluster CL1232-1250 at $z=0.54$ (top row) and cluster CL1216-1201 at $z=0.79$ (bottom row). The images are $\sim 2$.' $5 \times 2$ '. 5 .

center of the galaxy using the IRAF routine "imcenter"; (b) determines the maximum galaxy semi-major axis length $\left(a_{\max }\right)$ out to which ellipses will be fitted by finding where the galaxy isophotes reach the sky level; (c) executes "ellipse" for a maximum number $N$ of iterations, for each object, analyzing each output on the fly to guide the next fit. The wrapper stops either if all the isophotes can be fitted or if the maximum $N$ of iterations is reached, where $N$ is typically set to be 300 . A fit is considered to be successful if all the isophotes can be fitted either in one single fit or via a combination of partial fits. The fitting process for an individual galaxy can fail completely if the center of an object cannot be found or if the surface brightness oscillates too strongly across the galaxy. This typically happens when a foreground star is present or when two objects overlap. For only $\sim 7 \%$ of objects in our initial sample, the ellipse fit failed and successful fits were obtained for 1906 galaxies. When using the IRAF task "ellipse" for ellipse fits, the goodness of the best-fit solution is measured by four harmonic amplitudes (A3, A4, B3, B4), which describe by how much the true isophote differs from the best-fit model ellipse (e.g., Jedrzejewski 1987). We find that the deviations from ellipses are typically small $(<10 \%)$.

Based on these fits, radial profiles of the surface brightness, $\epsilon$, and PA are derived, and the fitted ellipses are overplotted onto the galaxy images. Examples of these profiles and overlays are shown in Figs. 4-6. These representations and the true images are the primary tools for the classification. In a first step, the disk inclination ( $i$ ) is determined using the $\epsilon$ profile. For each galaxy, an interactive visualization tool (Jogee et al. 2004) is used to display the overlay and radial profiles, interactively determine the disk parameters (ellipticity, PA) and bar parameters (maximum ellipticity, semi-major axis, PA), and assign the main classification of "inclined", "unbarred", and "barred". If the disk inclination, i.e., the inclination in the outer parts of the galaxy is higher than $60^{\circ}$ (or $\epsilon>0.5$ ) the galaxy is classified as being too inclined and excluded from further analysis. The galaxy shown in Fig. 4 is such an object. We find that $\sim 34 \%$ (652 objects) of the sample galaxies have $i>60^{\circ}$, which is similar to results for other samples (Jogee et al. 2004; Barazza et al. 2008), that are comparable in size and where the same method has been applied.

The remaining galaxies were then classified as unbarred or barred, based on the following quantitative criteria: (1) $\epsilon$ increases steadily to a global maximum higher than 0.25 , while the PA value remains constant (within $10^{\circ}$ ); and (2) $\epsilon$ then drops by at least 0.1 and the PA changes at the transition between the bar to the disk region. Figure 6 shows a galaxy, which meets these two criteria. While unbarred spiral galaxies can also reach large $\epsilon$ and exhibit prominent drops in their $\epsilon$ profiles in the region dominated by spiral arms, this is always accompanied by strong isophotal twists. Since we exclude too inclined galaxies, criterion (2) is characteristic of a barred disk, because the disks are typically more circular than the bars for moderately inclined galaxies. After classifying a galaxy, we used our interactive display tool to measure the ellipticity, PA, and semi-major axis of its outer disk. For galaxies classified as barred, we measure the same quantities, as well as the maximum ellipticity, $e_{\text {bar }}$, of the bar and the semi-major axis, $a_{\max }$, of maximum bar ellipticity. We use $e_{\text {bar }}$ as a partial measure of the bar strength and $a_{\max }$ as an estimate for the semi-major axis of the bar, $a_{\mathrm{bar}}$. A detailed theoretical and empirical justification of this approach is provided in Marinova \& Jogee (2007) and Menéndez-Delmestre et al. (2007) (see also the discussion in Sect. 5).

The classifications and quantities measured are based on the observed images and profiles and are therefore affected by projection effects (for a detailed discussion of how disk inclinations affect apparent bar sizes, see Barazza et al. 2008). We did not attempt to deproject our galaxies, since it is difficult to determine the PA in the outer disks accurately enough, particularly for galaxies at high redshifts. The resulting large errors in the PA would cause inaccurate deprojections. We also note that the statistical results before and after deprojection are very similar (Marinova \& Jogee 2007).

Of the remaining 1254 moderately inclined $\left(i<60^{\circ}\right)$ galaxies, we exclude another 309 objects for two reasons: $i$ ) the presence of a close neighbor, whose outer isophotes overlap with the target galaxy, can cause "ellipse" to fit the two galaxies simultaneously. The number of these cases is relatively high, due to the fact that many galaxies in our sample are located in or close to cluster centers, where the galaxy density is high; $i$ ) galaxies with very low surface brightnesses, resulting in very messy profiles, which could not be properly classified. Our final sample therefore comprises 945 disk galaxies, among which we find 238 to be barred. 

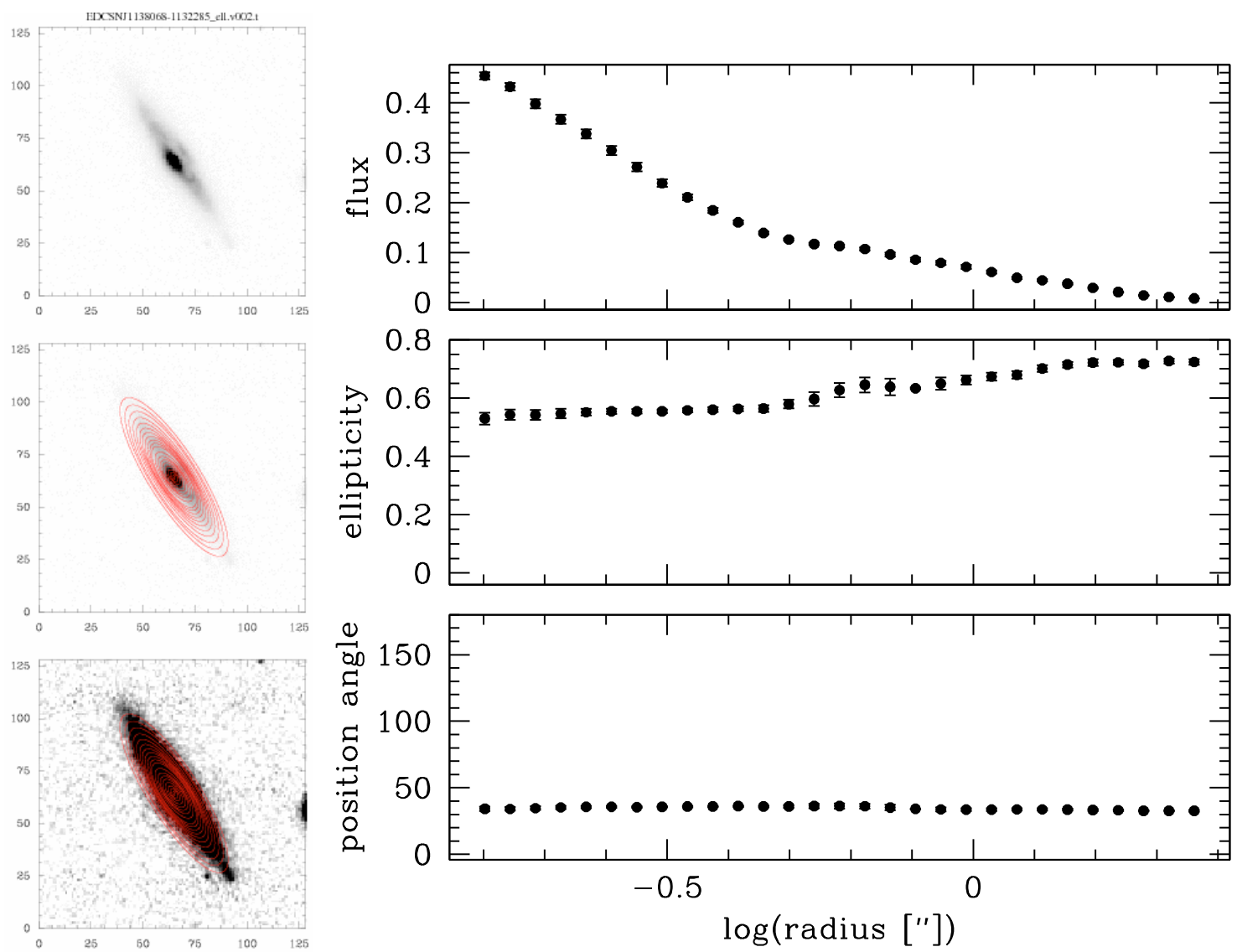

Fig. 4. This is an example of an inclined $\left(i>60^{\circ}\right)$ galaxy, identified from the overlays and radial profiles that are generated by the ellipse fits. Such galaxies are excluded from our final sample. Left: the top image shows only the galaxy, while the middle and bottom images show the ellipses overlaid on the galaxy, with different greyscale stretches to emphasize the inner and outer regions of the galaxy. The images are roughly $6^{\prime \prime}$ on a side. Right: the radial profiles of surface brightness (top), ellipticity $\epsilon$ (middle), and PA (bottom) are shown. In the outer parts of the galaxy, the PA is flat and the ellipticity is fairly constant at $\epsilon>0.5$, showing that the galaxy has a high inclination $\left(i>60^{\circ}\right)$.

\subsection{The detectability of bars}

As shown by several studies (e.g., Knapen 1999; Eskridge et al. 2000; Marinova \& Jogee 2007), the detectability of bars improves at longer wavelengths. The bar fraction measured for near-infrared observational studies are typically slightly higher than for studies based on optical imaging. The main reason for this difference is extinction caused by dust absorption, which is less severe at longer wavelengths. Our sample of disk galaxies covers the redshift range $z=0.4-0.8$, where the $I$-band observations correspond to rest-frame $B$ to $V$. Hence, band-shifting effects should not be so strong, but since the rest-frame range is rather blue, we should miss bars due to dust absorption, and the provided bar fractions must be considered as lower limits. In addition, enhanced star formation and dust obscuration further impede bar detection at higher redshifts.

Another factor affecting the identification of bars in disk galaxies is the resolution of the observations. We apply two requirements for bar detection: (1) a bar can only be detected if its angular diameter encompasses at least 4 PSFs; (2) the bar size (i.e., the bar radius, $a_{\mathrm{bar}}$ ) covers at least four pixels. Criterion (1) is based on the fact that we need at least one PSF for the bulge, two PSFs for the bar region, and one PSF for the disk beyond the bar. This resolution is needed to detect reliably the quantitative bar signatures described in Sect. 3.1. The PSF on our images is 0 .'09, which corresponds to $\sim 675 \mathrm{pc}$ at the highest redshift $(z \sim 1.0)$ and 4 PSFs correspond to $\sim 2.7 \mathrm{kpc}$ at that redshift.
In Fig. 7, we plot redshift versus the bar diameter in arcsec. The dotted line indicates 4 PSFs (i.e., 0!'36). The second requirement for bar detection is illustrated in Fig. 8, where we plot $a_{\text {bar }}$ as a function of redshift. The dotted line indicates the lower limit for large-scale bars at $1 \mathrm{kpc}$ (Laine et al. 2002) and the dashed line corresponds to the absolute size of four pixels. For increasing redshifts higher than $\sim 0.4$, we begin to lose the smallest bars. However, based on both bar-detection criteria, we are complete for bars with $a_{\mathrm{bar}} \gtrsim 2 \mathrm{kpc}$.

Besides resolution, other factors, such as increased obscuration due to both dust and star formation and surface-brightness dimming, can prevent bar detection at higher redshifts. Small bars are particularly affected by these factors. We point out that all results presented in this study have been checked with regard to a possible bias with respect to redshift (e.g., see Fig. 13).

\section{The optical bar fraction and its dependence on galaxy properties}

In the following, we discuss the properties of our disk galaxy sample based on our bar classification. We show how the presence of a bar is related to other characteristics of the galaxies and whether there is a difference between galaxies in clusters and the field. In general, we use the sample of 945 moderately inclined disk galaxies for our analysis, while when comparing cluster and field galaxies we restrict the sample to objects with spectroscopic observations (136 galaxies in clusters and 105 galaxies 

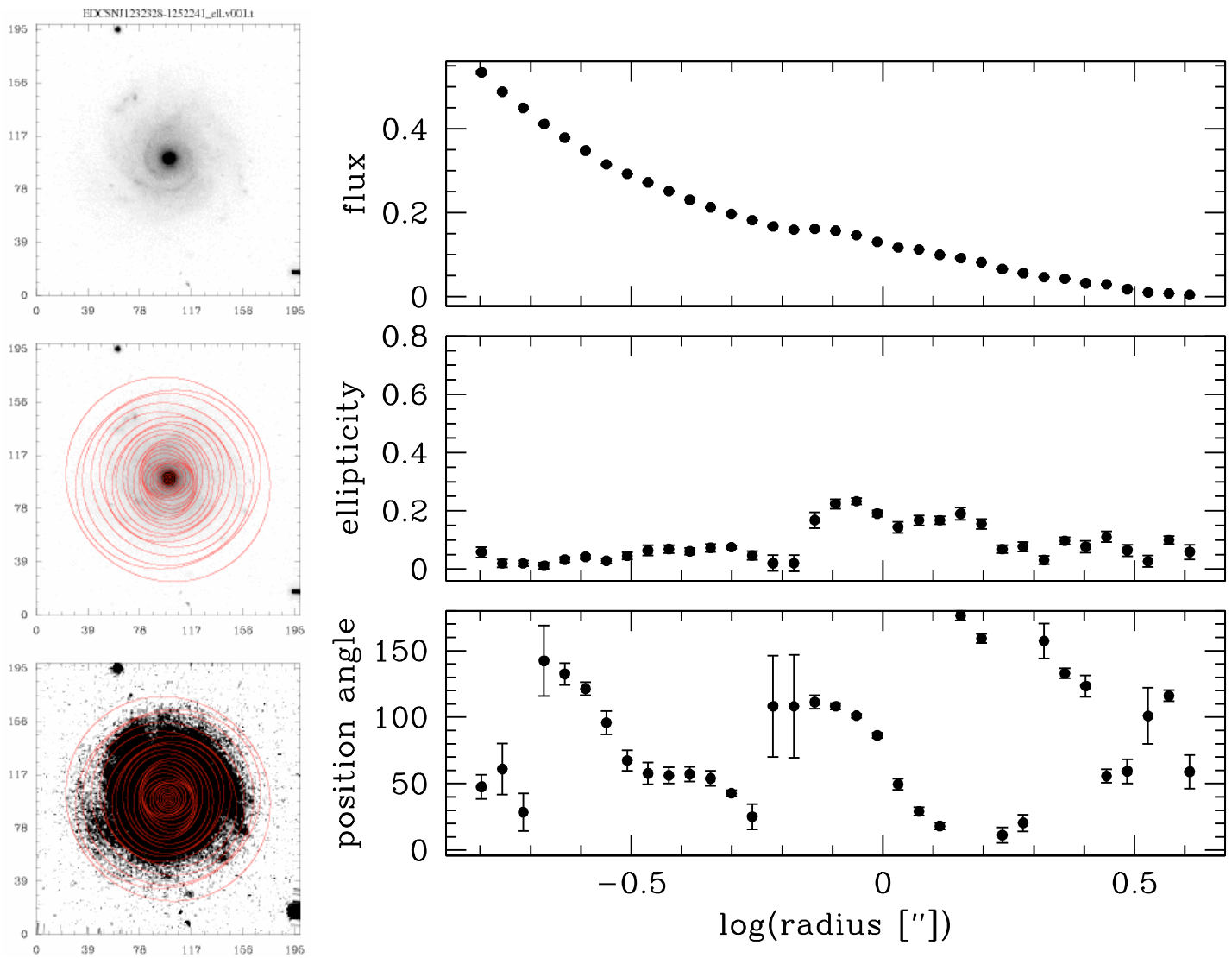

Fig. 5. The same as Fig. 4, but for a galaxy classified as unbarred according to our ellipse fits. The images are roughly $10^{\prime \prime}$ on a side.
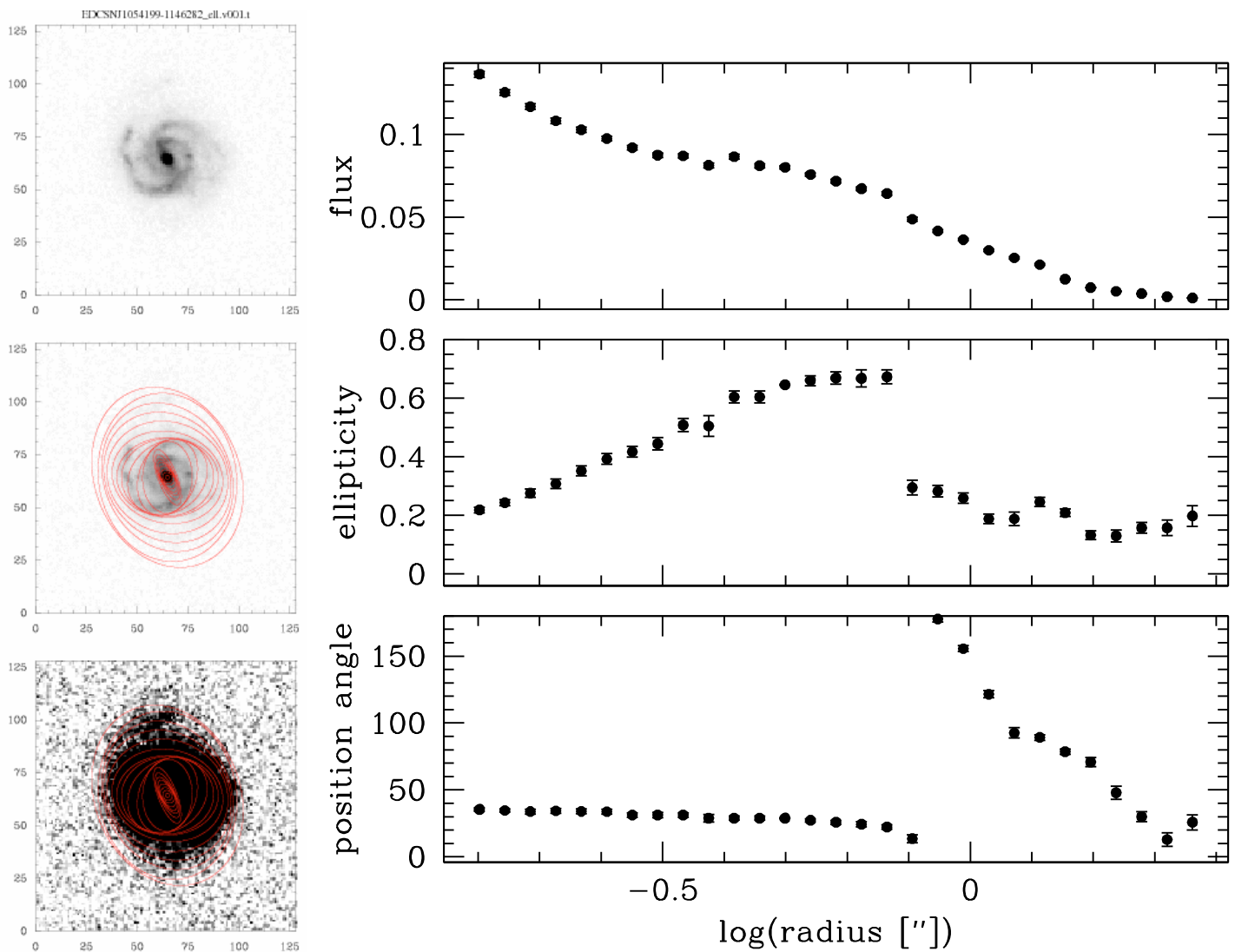

Fig. 6. The same as Fig. 4, but for a galaxy classified as barred from ellipse fits. The images are roughly 6" on a side. Over the bar region, $\epsilon$ rises smoothly to a global maximum of $\sim 0.7$, while the PA remains approximately constant. After the end of the bar at the transition to the more circular disk, the ellipticity decreases sharply at $\sim 0{ }^{\prime} 8$ and the PA starts to change significantly at this point. 


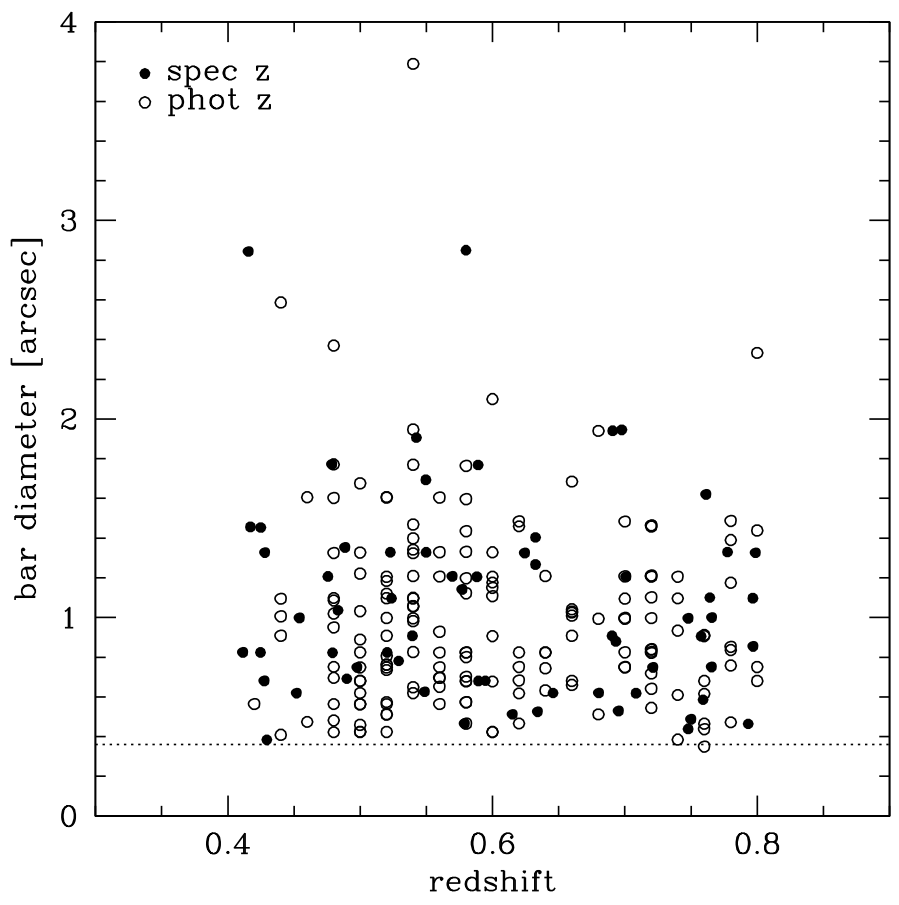

Fig. 7. Plot showing the bar diameter in arcsec versus redshift. The filled points indicate spectroscopically based redshifts, while open points are for photometric redshifts. The dotted horizontal line marks 4 PSFs (i.e. 0!'36).

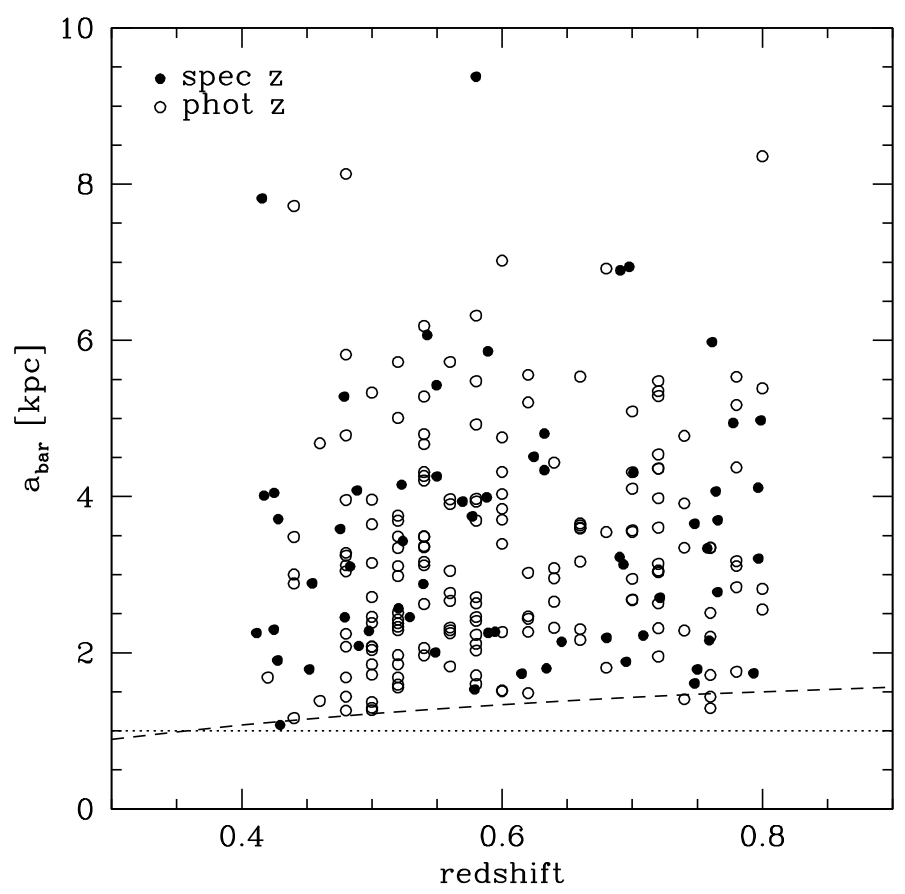

Fig. 8. The same as Fig. 7, but this time showing the bar size in terms of bar semi-major axis $a_{\text {bar }}$. The dotted horizontal line shows the separation between large scale bars $\left(a_{\mathrm{bar}}>1 \mathrm{kpc}\right)$ and nuclear bars $\left(a_{\mathrm{bar}} \leq 1 \mathrm{kpc}\right)$. The dashed line indicates the absolute size of four pixels.

in the field). This reduces the size of the sample considerably, but ensures reliable membership assignment. In particular, the contamination of the cluster sample by field galaxies based on photometric redshifts impedes an accurate separation between cluster and field galaxies. The error bars in the following plots only include Poissonain errors. Specific factors affecting bar detection are discussed in Sect. 3.2.

Among the 945 disk galaxies in our sample, we find 238 to be barred, and hence derive an optical bar fraction $\left(f_{\mathrm{bar}}\right)$ of $\sim 25 \%$. This is significantly lower than is typically found in optical studies of local galaxies (Eskridge et al. 2000; Marinova \& Jogee 2007; Reese et al. 2007; Barazza et al. 2008; Aguerri et al. 2009), but in good agreement with studies at intermediate redshifts (Elmegreen et al. 2004; Sheth et al. 2008). This could indicate that the bar fraction is lower at higher redshifts, which would imply that we can detect nearly all bars in our sample. Possible other reasons for the difference compared to local studies have been given in Sect. 3.2. We also point out that the bar fraction for bulge-dominated galaxies is found to be significantly lower than for disk-dominated systems in studies of local galaxies (Barazza et al. 2008; Aguerri et al. 2009), a result confirmed by our own study (Sect. 4.1). Since our sample is dominated by early-type disks ( $>74 \%$ are earlier than $\mathrm{Sc}$ ), a relatively low bar fraction might be expected. If we only consider strong bars $(e>0.4)$, we obtain a bar fraction of $\sim 20 \%$, which also agrees well with earlier studies (Jogee et al. 2004). For the spectroscopically confirmed cluster sample, we obtain $f_{\text {bar }}=24 \%$ (136 objects/33 bars), and for the corresponding field sample, we derive $f_{\text {bar }}=29 \%(105 / 30)$. These values agree within the uncertainties with the result for the complete sample and indicate that the frequency of bars in clusters is almost identical to that in the field. This finding indicates that bar formation, in general, is independent of environment, which was also found by van den Bergh (2002). On the other hand, Varela et al. (2004) found twice as many bars in perturbed as in isolated galaxies. We investigate these results further in Sect. 7.

\subsection{The bar fraction as a function of morphological type}

In Fig. 9, we show the optical bar fraction as a function of Hubble type. The results for the total sample (Figs. 9a and b) indicate that the bar fraction increases towards later Hubble types. Galaxies earlier than Sb have bar fractions below 20\%, while all later types exhibit higher bar fractions. The monotonically increasing bar fraction along the sequence and the significant difference between those of S0s $\left(f_{\text {bar }}=8 \pm 8 \%\right)$ and Scs $\left(f_{\text {bar }}=36 \pm 5 \%\right)$ indicate that the effect is significant. Our results for $\mathrm{Sd}$ and $\mathrm{Sm} / \mathrm{Im}$ types are less robust due to the smaller number of galaxies with these morphologies. Similar analyzes (Odewahn 1996; Elmegreen et al. 2004) based on the Third Reference Catalog of Bright Galaxies (de Vaucouleurs et al. 1991), however, obtained different results. Their bar fraction was at its lowest for Sc galaxies and increased towards earlier and later types. We cannot compare the results for $\mathrm{S} 0$ galaxies, since these wre not included in the earlier investigations. On the other hand, our result is consistent with two studies based on the Sloan Digitized Sky Survey (SDSS), where, however, no Hubble types were available: Barazza et al. (2008) showed that the bar fraction is significantly higher for disk-dominated galaxies than for galaxies hosting prominent bulges. Although the sample in that study was dominated by late-type disks, the connection found between the prominence of the bulge and the bar fraction can also be inferred from our result. In their analysis of bars in local disk galaxies, Aguerri et al. (2009) found that S0 galaxies have a significantly lower bar fraction (by $\sim 23 \%$ ) than galaxies of later types, which agrees well with our result. A somewhat different result was reported by Sheth et al. (2008), who found a slight preference for bars in bulge-dominated systems at high redshifts. We emphasize that the presence of a large bulge should not 

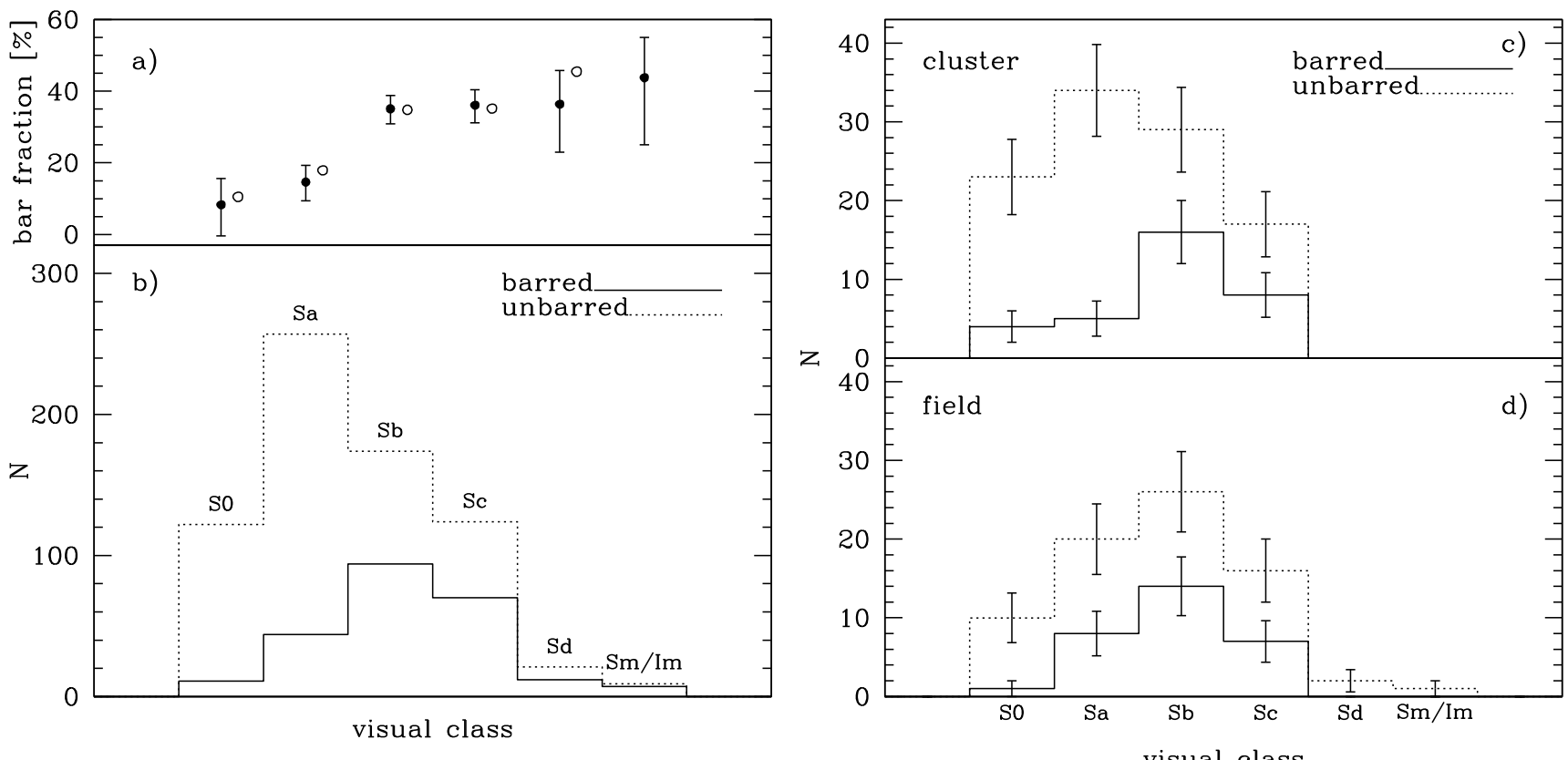

Fig. 9. The distribution of barred and unbarred disk galaxies as a function of Hubble type. a) The bar fraction as a function of Hubble type. The filled circles show the relation for our final sample of 945 disk galaxies. The open circles show the relation for the complete sample with $M_{V} \leq-20$. b) Histograms of barred (solid line) and unbarred (dotted line) disk galaxies. c) The same as b), but for the spectroscopically based subsample of cluster members. d) The same as b), but for the spectroscopically based subsample of field galaxies.
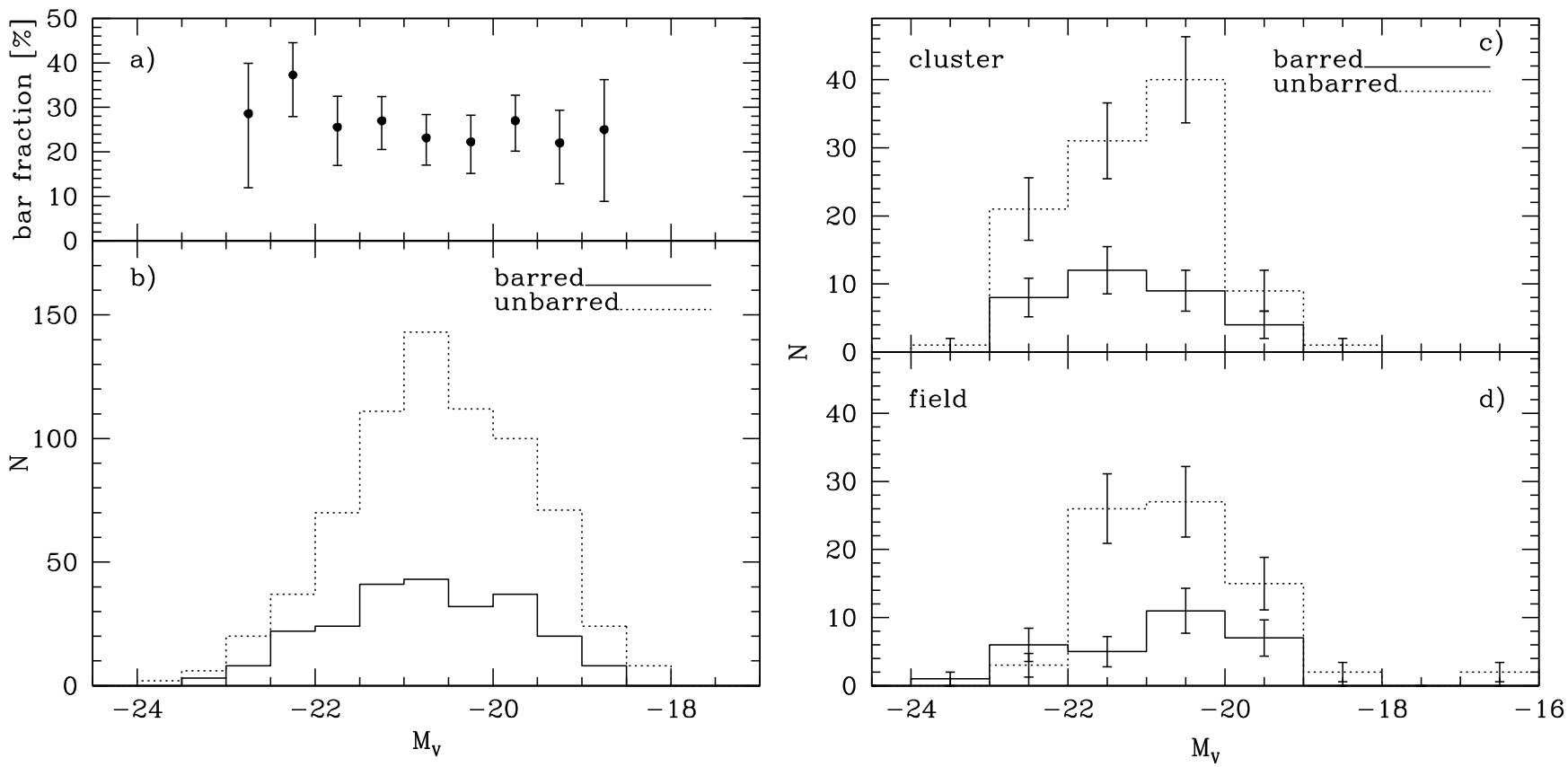

Fig. 10. The same as Fig. 9, but for the absolute $V$ magnitude. In a) only bins with more than 25 objects are shown.

impede bar detection, since we are only interested in large-scale bars and exclude strongly inclined objects, for which a massive bulge could make bar detection difficult.

Figures 9c and d show the same relations for our spectroscopic sample. The distributions indicate that early-type disks are more prominent in clusters, as expected, whereas the bar fractions in the field and in clusters are quite similar. On the other hand, we find differences in the bar fractions for individual Hubble types in clusters and the field, for instance for Sa galaxies (cluster: 13\%, field: 29\%). However, due to the small number of objects involved (20-40 galaxies), the significance of this result cannot be assessed reliably.

\subsection{The bar fraction as function of magnitude and color}

In Fig. 10, we show how the optical bar fraction depends on the rest-frame total $V$-band magnitude of the galaxy, for both the full sample and the spectroscopic subsample. For cluster members, the rest-frame magnitudes are calculated based on the cluster redshift (as opposed to the galaxy redshift). The bar fraction 


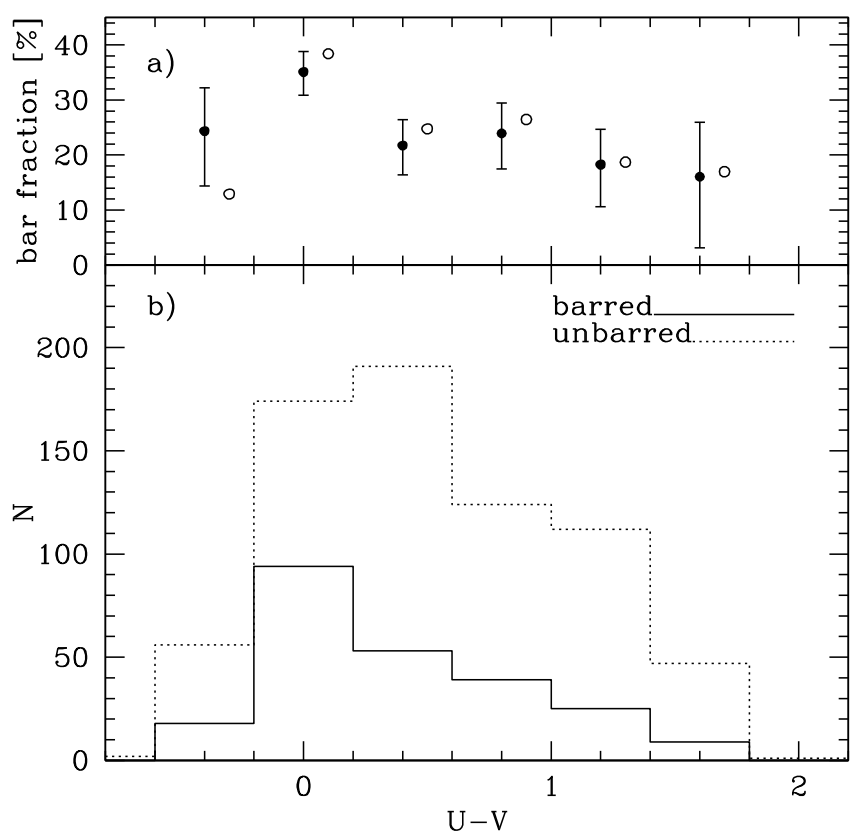

Fig. 11. The same as Fig. 10, but for rest-frame $U-V$ color.

Table 2. Bar fractions per Hubble type for bright and faint subsamples.

\begin{tabular}{ccc}
\hline \hline $\begin{array}{c}\text { Hubble } \\
\text { type }\end{array}$ & $\begin{array}{c}\text { Bar fraction (Number of objects) } \\
<-21.0 \mathrm{mag}\end{array}$ & $\geq-21.0 \mathrm{mag}$ \\
\hline $\mathrm{S} 0$ & $14 \%(44)$ & $6 \%(89)$ \\
$\mathrm{Sa}$ & $21 \%(121)$ & $11 \%(180)$ \\
$\mathrm{Sb}$ & $40 \%(117)$ & $31 \%(151)$ \\
$\mathrm{Sc}$ & $32 \%(59)$ & $38 \%(135)$ \\
\hline
\end{tabular}

Notes: the table shows results for 896 objects in the range $0.4<z<0.8$. (The $52 \mathrm{Sd} / \mathrm{Sm} / \mathrm{Im}$ galaxies are not considered in this table.) The samples are split at $M_{V}=-21.0 \mathrm{mag}$, which is the mean $V$-band magnitude of the total sample.

remains relatively constant with galaxy magnitude. The slight decrease towards lower magnitudes lies within the uncertainties and is therefore insignificant. Early-type disks are generally more luminous than late-type disk and have a lower bar fraction (Fig. 9). We may therefore naively expect that the bar fraction should increase toward lower magnitude. This trend could be erased, however, if the brighter galaxies in each morphological class had a higher bar fraction, which has been found in a study of barred disks in the Abell 901/902 supercluster environment (Marinova et al. 2009). To investigate this possibility, we split the morphological subsamples at $M_{V}=-21.0 \mathrm{mag}$, which is the mean $V$-band magnitude of the complete sample $\left(M_{V} \leq-20.0\right)$, and determine the bar fractions for the brighter and fainter parts of these subsamples. The result is shown in Table 2. For Hubble types $\mathrm{S} 0-\mathrm{Sb}$, the bright subsample does indeed have a higher bar fraction than the faint subsample. For Sc disks, the trend is, however, reversed. This explains the almost constant bar fraction as a function of magnitude. The result indicates that most bars in early-type disks have a relatively high surface brightness and therefore contribute significantly to the high luminosity.

Figures $10 \mathrm{c}$ and d show the distributions for the cluster and field subsamples. The cluster sample is slightly brighter than the field sample, but in terms of bar fraction they are almost identical.

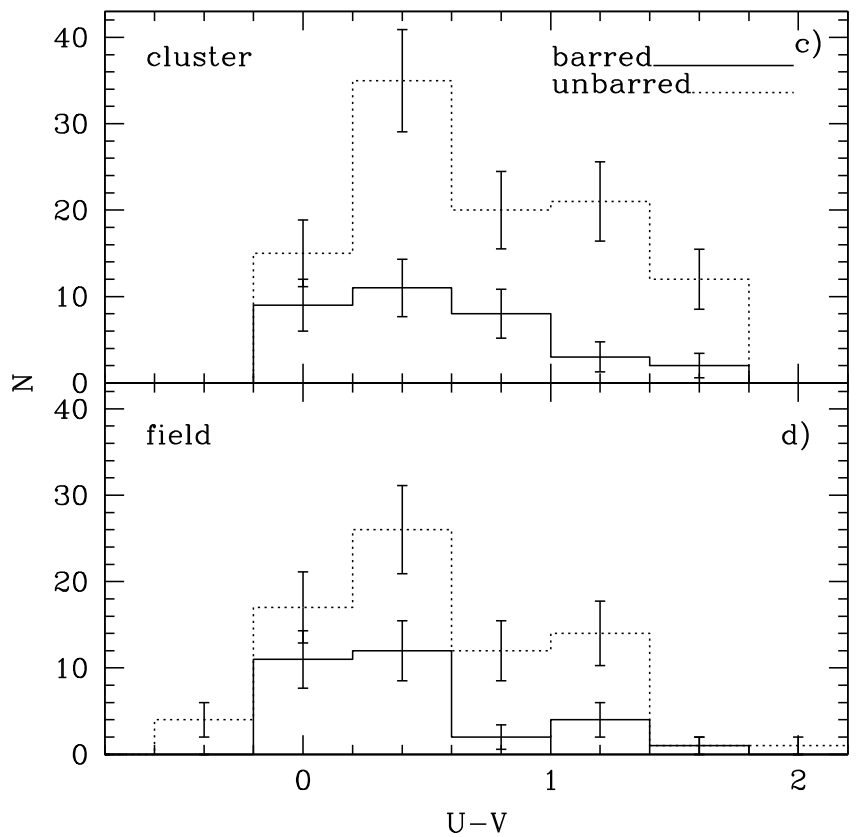

The corresponding relations with respect to rest-frame $U-V$ color are shown in Fig. 11. The bar fraction declines toward redder colors (Figs. 11a and b), as expected from the relations found in terms of morphology. This finding emphasizes that late-type disks are more likely to host bars than early-type disks. The same trend is also found for the cluster and field subsamples (Figs. 11c and d), although the cluster sample is on average significantly redder than the field sample.

\subsection{The bar fraction as a function of effective radius}

The effective radius $\left(r_{\mathrm{e}}\right)$ determined by applying a Sérsic fit to the entire galaxy is a measure of the concentration of the galaxy light and a partial measure of the prominence of the bulge. A relation between Hubble type and $r_{\mathrm{e}}$ is therefore expected and also found in our sample. We computed the mean effective radius for each Hubble type and obtain the following result: S0: $\left\langle r_{\mathrm{e}}\right\rangle=1.81 \mathrm{kpc} ; \mathrm{Sa}:\left\langle r_{\mathrm{e}}\right\rangle=2.46 \mathrm{kpc} ; \mathrm{Sb}:\left\langle r_{\mathrm{e}}\right\rangle=3.54 \mathrm{kpc} ; \mathrm{Sc}:$ $\left\langle r_{\mathrm{e}}\right\rangle=4.27 \mathrm{kpc}$; and $\mathrm{Sd}:\left\langle r_{\mathrm{e}}\right\rangle=3.61 \mathrm{kpc}$. However, since $r_{\mathrm{e}}$ only indicates the distribution of the light in the galaxies, it is more related to the concentration of the light than the Hubble type. Figure 12 shows the bar fraction as a function of $r_{\mathrm{e}}$. There is a continuous rise in the bar fraction with increasing $r_{\mathrm{e}}$ (Fig. 12a). This again seems to be just another representation of the effect already indicated in the relations based on morphology and color. Galaxies with larger central light (or mass) concentrations have less bars than disk-dominated galaxies with small central objects. The result also agrees with the findings of Barazza et al. (2008) where a similar plot is shown and with the results of Hernández-Toledo et al. (2008), which are based on the light concentration index. However, the apparent lack of the smallest bars at higher redshifts might contribute to this result. This could be the case if galaxies with small effective radii tend to have shorter bars. We therefore reproduce Fig. 12a for three different subsamples. The result is shown in Fig. 13. The solid line is for galaxies at $z \leq 0.60$ (the median redshift of the sample), the dotted line is for galaxies at $z>0.60$, and the dashed line shows the relation assuming that only bars with $a_{\text {bar }}>3 \mathrm{kpc}$ 

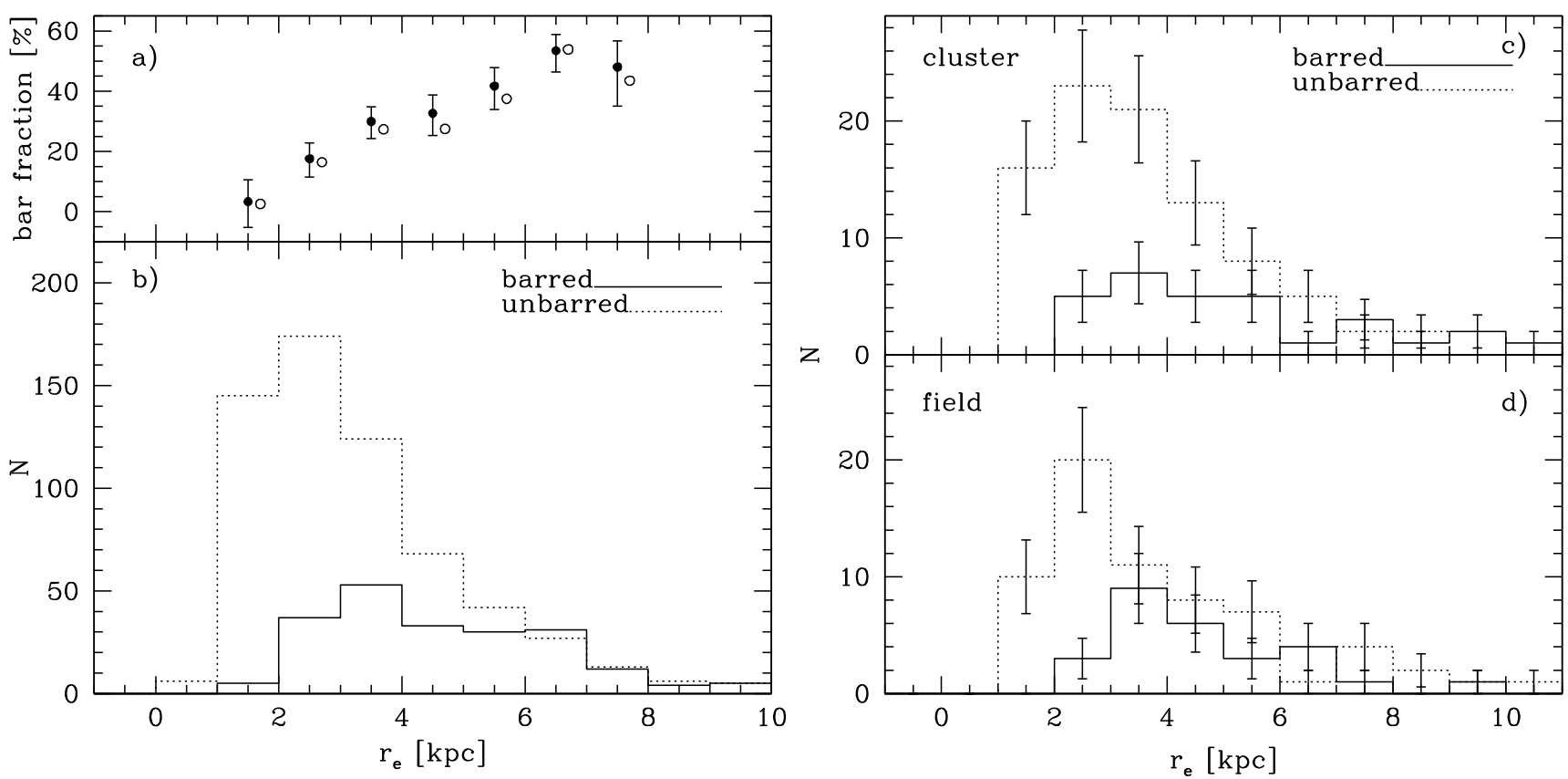

Fig. 12. The same as Fig. 10, but for the effective radius determined from a Sérsic fit. Galaxies in the clusters cl1227.9-1138 and cl1227.9-1138a are excluded, since Sérsic fits are unavailable.

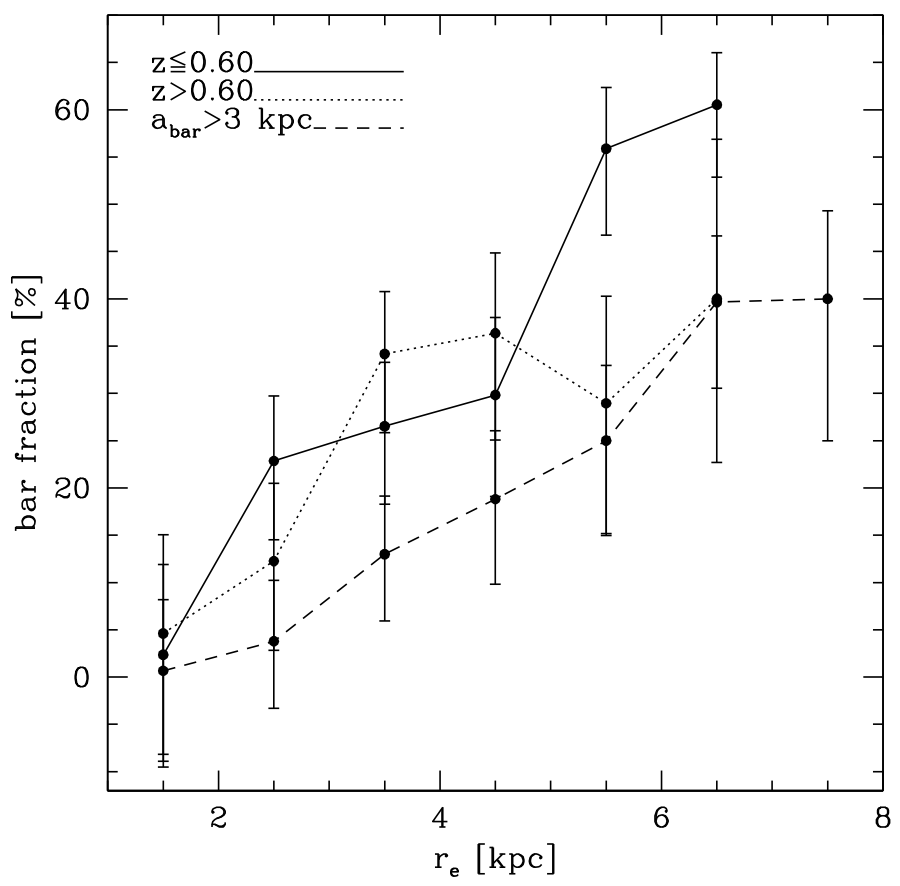

Fig. 13. The same as Fig. 12a, but for three different subsamples: the solid line is for galaxies at $z \leq 0.60$ (the median redshift of the sample), the dotted line is for galaxies at $z>0.60$, and the dashed line shows the relation assuming that only bars with $a_{\mathrm{bar}}>3 \mathrm{kpc}$ can be detected.

can be detected (i.e., all bars with $a_{\text {bar }}<3 \mathrm{kpc}$ are considered to be unbarred in this case). All subsamples clearly show that the bar fraction increases towards galaxies with larger effective radii. We can therefore conclude that the result is real and not caused by the lower bar detection rate at higher redshift.

Finally, the same result is again found in the two subsamples in Figs. 12c and d. This finding supports the assumption that the presence of a bar is related to the disk structure and the magnitude of the central mass concentration.

\section{The properties of the bars}

The two main properties of bars typically measured and analyzed in bar studies are the bar size and strength. Different methods are adopted to determine these quantities. In particular, the bar strength can be measured using the gravitational torque exerted by the bar (Block et al. 2002; Buta et al. 2005), the maximum ellipticity of the bar (Martin 1995; Jogee et al. 1999; Knapen et al. 2000; Laine et al. 2002; Marinova \& Jogee 2007; Barazza et al. 2008), Fourier decomposition (Elmegreen et al. 1996), and visual estimates of strength (Eskridge et al. 2002). As described in Sect. 3.1, our measurements are based on ellipse fitting. Since this fitting provide the basis of the classification, the determination of the maximum ellipticity $\left(e_{\mathrm{bar}}\right.$, interpreted as the bar strength) and the radius ( $a_{\mathrm{bar}}$, interpreted as the bar size), at which this maximum occurs, is straightforward. However, we emphasize that the maximum bar ellipticity is only a partial measure of the bar strength, because it provides no indication of the mass of the bar. However, Shen \& Sellwood (2004) demonstrated that the maximum ellipticity correlates strongly with the relative amplitude of the bisymmetric Fourier component of the mass density averaged over a certain inner radial range, where the bar dominates. In addition, the gravitational torque, on average, correlates with the maximum ellipticity of the bar (Laurikainen et al. 2002). We can therefore regard the bar ellipticity to some extent as a measure of the bar strength.

In Fig. 14, we show the bar size and bar ellipticity distributions. A significant majority ( $86 \%$ ) of bars have sizes $\leq 5 \mathrm{kpc}$, in agreement with un-deprojected results in earlier studies (Jogee et al. 2004; Marinova \& Jogee 2007; Barazza et al. 2008). Similarly, the distribution of $e_{\mathrm{bar}}$ is consistent with these earlier studies. Figure 15 shows the corresponding distributions for the cluster and field subsamples. The cluster sample exhibits no prominent peak in the bar size distribution (left panel), but is slightly skewed towards larger sizes. In contrast, the field sample shows a similar distribution to that of the total sample. Hence, bars in clusters tend to be longer than bars in field galaxies. 


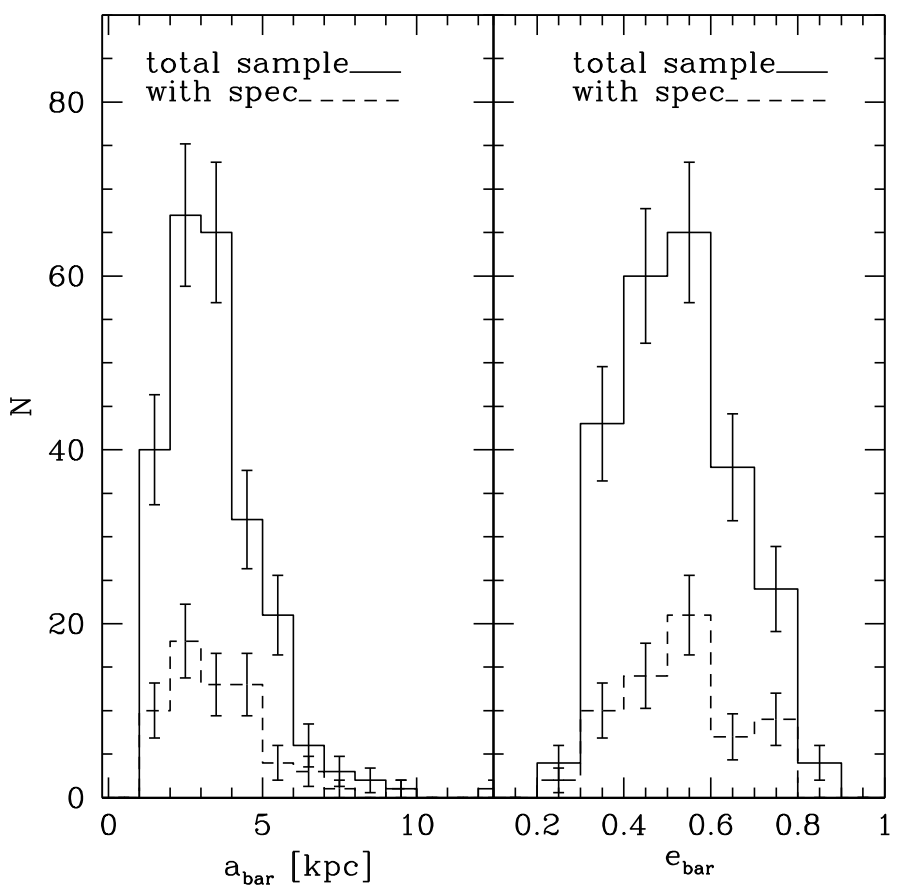

Fig. 14. The bar size (left) and bar ellipticity (right) distributions for the total sample (solid lines) and the spectroscopic sample (dashed line).

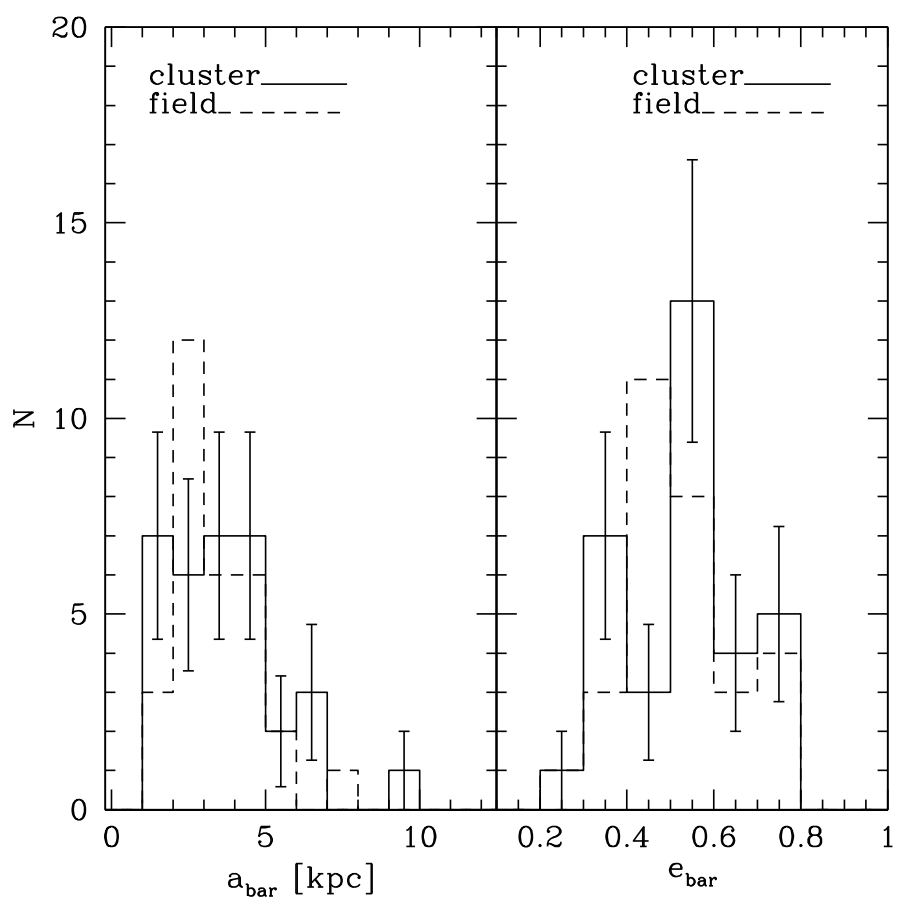

Fig. 15. The same as Fig. 14, but only showing the spectroscopically defined cluster (solid lines) and field (dashed lines) subsamples. For readability, error bars are only shown for the cluster sample.

The differences that we observe between the bar size distributions of the field and cluster samples (Fig. 15) could indicate that the cluster environment has an impact on the bar sizes. In fact, numerical simulations show that tidally induced bars are on average longer than bars in isolated disks (Aguerri \& GonzálezGarcía 2009). To investigate this possibility, we measured the projected distances $\left(R_{\mathrm{CC}}\right)$ of the cluster galaxies from the corresponding cluster center, assuming that all galaxies are at the cluster redshift. This value was then normalized by $R_{200}$, the

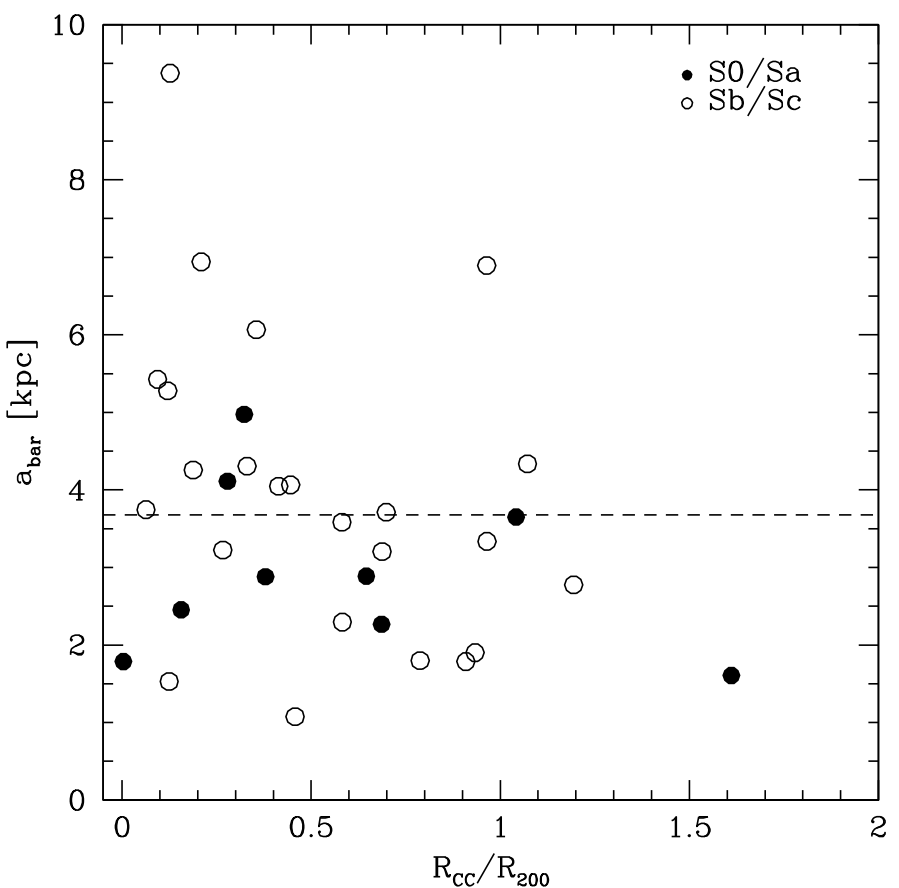

Fig. 16. The bar size as a function of normalized clustercentric distance for the spectroscopic cluster subsample. Filled points are for $\mathrm{S} 0 / \mathrm{Sa}$ types and open point for $\mathrm{Sb} / \mathrm{Sc}$ types. There are no other Hubble types in the sample. The dashed line indicates the mean bar size for this sample of $3.68 \mathrm{kpc}$.

radius within which the average mass density is equal to 200 times the critical density. We use the definition given in Finn et al. (2005, Eq. (8)). In Fig. 16, we plot the bar size versus the normalized clustercentric distance. Larger bars tend to be located close to the cluster center. The majority of bars larger than the average size of $3.68 \mathrm{kpc}$ (indicated by the dashed line) are located within $R_{\mathrm{CC}} / R_{200}<0.5$. A KS-test indicates that there is a $\sim 1 \%$ probability that the $a_{\text {bar }}>3.68 \mathrm{kpc}$ sample stems from the same distribution as the complementary sample of small bars in terms of $R_{\mathrm{CC}} / R_{200}$. We must remember that we are using projected distances, although this is unlikely to explain the trend entirely. It is also interesting that most of the $a_{\mathrm{bar}}>3.68 \mathrm{kpc}$ bars are found in disks of intermediate Hubble type. However, we highlight that the number of objects (33) in the plot is small and the significance of this finding is difficult to assess. We will nevertheless discuss possible origins of this effect in Sect. 7.

In Fig. 17, we show how the bar properties are related to Hubble type. The two panels show the mean bar size and bar ellipticity for each Hubble type. The mean bar sizes are in the range $2.5-3.5 \mathrm{kpc}$. For early-type disks $(\mathrm{S} 0-\mathrm{Sb})$, this is consistent with the results of Erwin (2005), whereas the mean bar sizes in late-type disks are larger than those measured by Erwin (2005). However, the number of objects in the Sd and $\mathrm{Sm} / \mathrm{Im}$ classes is rather small in our sample (see Fig. 9). On the other hand, there is an obvious relation between morphological type and mean bar ellipticity, which increases by more than 0.2 from early to late types. However, this result is at least in part due to the measurement including the bulge, which can lead to an underestimation of the bar ellipticity (Gadotti 2008). The measured bar ellipticity stems from the outermost isophote attributed to the bar. The bulge light contributes to the central part of this isophote. A large and luminous bulge will therefore widen this isophote at the center and, hence, lower the bar ellipticity. With the data available, it is impossible to estimate the impact that this effect could have on the relation in Fig. 17 


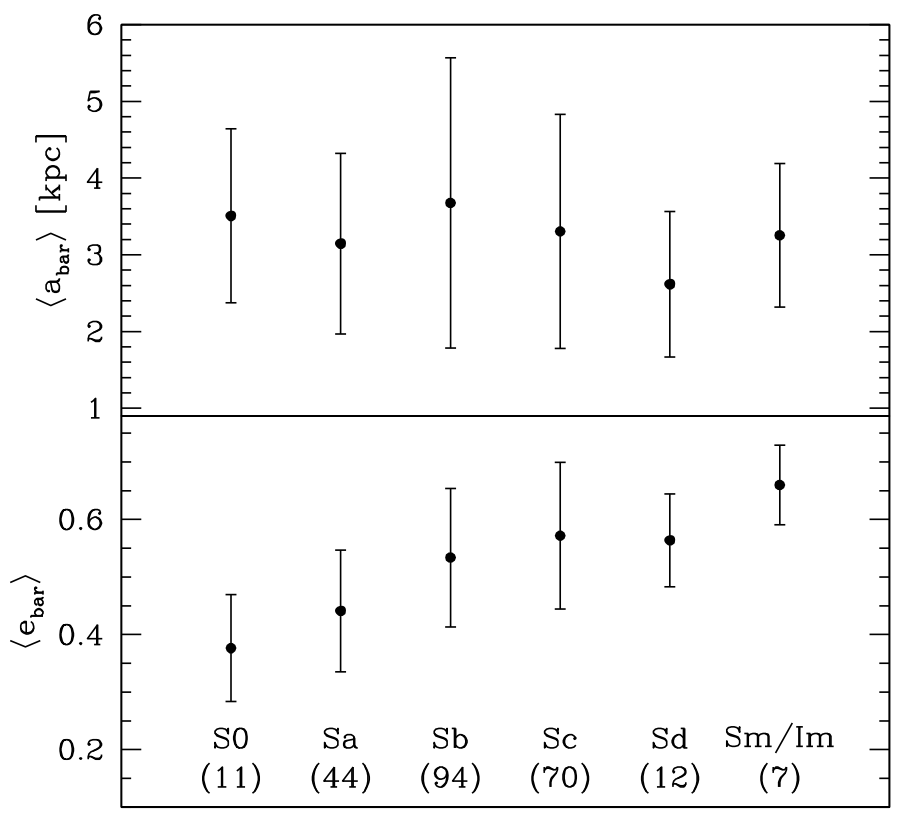

visual class

Fig. 17. The average bar size (top) and average bar ellipticity (bottom) as a function of Hubble type. The number in brackets gives the number of objects in that bin. The error bars indicate standard deviations of the mean.

(lower panel). An accurate bulge-bar-disk decomposition would be required to investigate the possibility that late-type disks indeed have stronger bars, on average. This is, however, beyond the scope of this analysis.

\section{The distribution of barred galaxies within the clusters}

In two studies of local galaxy clusters, evidence has been found that barred galaxies are preferentially located at the cluster core. An analysis of the clustercentric distances of barred galaxies in the Coma cluster (Thompson 1981) showed that a significantly larger fraction of barred galaxies are located at the cluster core than larger clustercentric distances (the adopted core radius for Coma was $\sim 784 \mathrm{kpc}$ ). In a similar study of the Virgo cluster, Andersen (1996) found that the barred disk galaxies are more centrally concentrated than the unbarred disks. For S0 galaxies, the same study found that the distributions of barred and unbarred objects are the same. In Fig. 18, we show the bar fractions as a function of the clustercentric distances (absolute and normalized). We find the highest bar fraction in the central bin. For the $R_{\mathrm{CC}}$ distribution, the bar fraction declines from $31 \%$ in the central bin to $18 \%$ at $\sim 1 \mathrm{Mpc}$. (The corresponding values for the complete sample are $30 \%$ and $15 \%$, respectively.) For the $R_{\mathrm{CC}} / R_{200}$ distribution, the corresponding values are $29 \%$ in the central bin and $22 \%$ at $R_{200}$. (The corresponding values for the complete sample are $30 \%$ and $21 \%$, respectively.) We emphasize again that the sample used is rather small, but we can safely say that barred galaxies do not avoid the cluster center. With the tendency for galaxy with large bars to be located close to cluster cores (Fig. 16), these findings indicate that regions of high galaxy density are favorable locations for bars.

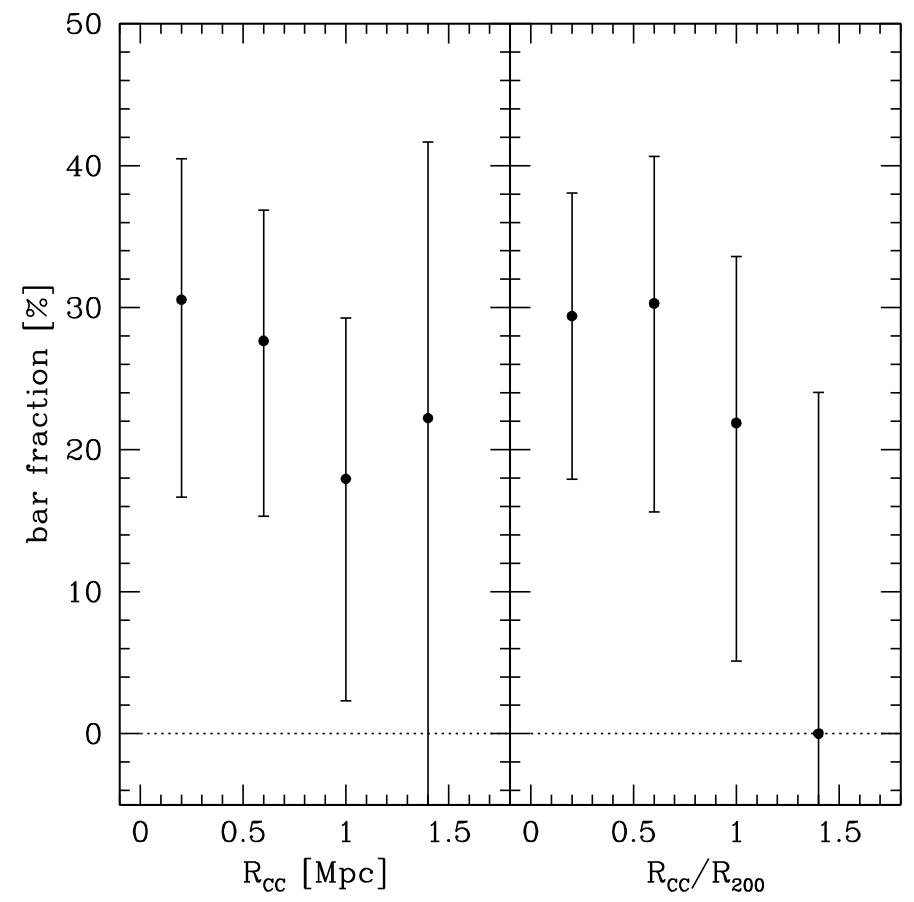

Fig. 18. The bar fraction as a function of the clustercentric distance (left) and the normalized clustercentric distance (right) for the spectroscopic subsample.

\section{Discussion}

We have found the optical bar fraction averaged over our entire sample covering the redshift range $z=0.4-0.8$ to be $\sim 25 \%$. The median redshift of the sample is 0.60 . In Sect. 3.2, we discussed how at these redshifts certain factors, such as reduced resolution, band shifting, enhanced obscuration by dust, could lead to a reduced bar detection rate. This could explain why the optical bar fraction of this sample is considerably lower than measured in local samples of disk galaxies (Marinova \& Jogee 2007; Barazza et al. 2008; Aguerri et al. 2009), but is in good agreement with studies at intermediate redshifts (Elmegreen et al. 2004; Jogee et al. 2004; Sheth et al. 2008). We note that all of these studies were based on samples primarily composed of galaxies in low density environments. A key question is whether the lower bar fraction at intermediate redshifts is primarily a result of the enhanced difficulty in identifying bars, or whether the number of barred disk galaxies was really lower at these redshifts. Several studies (Abraham et al. 1999; van den Bergh et al. 2000) claimed that the bar fraction was significantly lower at $z \gtrsim 0.5$. This was confirmed by Sheth et al. (2008). Only for a small subsample of large and massive galaxies was a constant bar fraction found (Sheth et al. 2003, 2008). In other studies, a fairly constant bar fraction out to $z \sim 1$ was found for strong bars (Jogee et al. 2004) or all bars (Elmegreen et al. 2004; Zheng et al. 2005). To determine the evolution in the bar fraction with redshift in our sample, we divided the sample into three redshift bins and measured the bar fraction and the mean bar sizes and ellipticities in each. The bin sizes were defined in order to obtain comparable numbers of objects in each bin. We only considered galaxies with spectroscopic redshifts. The corresponding results are shown in Table 3. The bar fraction was found to decline modestly from $30 \%$ at $0.4<z \leq 0.55$ to $22 \%$ at $0.7<z \leq 0.8$. The average bar sizes in the lowest and highest redshift bins are roughly the same, and the average bar strength does not change within our redshift range. This indicates that we have not missed significant 
Table 3. Bar fractions and properties as a function of $z$.

\begin{tabular}{cccc}
\hline \hline Spec- $z$ & $0.4<z \leq 0.55$ & $0.55<z \leq 0.7$ & $0.7<z \leq 0.8$ \\
\hline \# of objects & 87 & 78 & 76 \\
bar fraction & $30 \%$ & $26 \%$ & $22 \%$ \\
$\left\langle a_{\text {bar }}\right\rangle[\mathrm{kpc}]$ & 3.38 & 3.83 & 3.37 \\
$\left\langle e_{\text {bar }}\right\rangle[\mathrm{kpc}]$ & 0.54 & 0.53 & 0.52 \\
\hline
\end{tabular}

Notes: the bar fraction and average bar sizes and ellipticities in three spectroscopically based redshift bins.

numbers of short or weak bars at higher redshifts. These results suggest that either the bar fraction decreases with increasing redshift, which would imply that we can nearly detect all bars in our sample, or that the decline is caused by the growing difficulty in identifying bars at higher redshifts. Reasons for the latter were given in Sect. 3.2. A detailed analysis of this issue was beyond the scope of this study.

The other results presented in the previous sections can be divided into two categories: (1) general relations between bars and the properties of their host galaxies, independent of whether the galaxies are cluster members or in the field; (2) relations regarding the specific locations of barred galaxies within the clusters.

\subsection{Relating bar fraction and characteristics to host galaxy properties}

We have found additional evidence that the bar fraction is related to the morphological structure of the host galaxies, which agrees with the results reported in Odewahn (1996), Barazza et al. (2008), and Marinova et al. (2009). The bar fraction rises from early- to late-type disks, or in other words, from bulge-dominated galaxies to disk-dominated galaxies (Figs. 9a and 12a). This appears to indicate that bars in bulge-dominated disks are more likely to be dissolved. However, the processes responsible cannot have been important since $z=0.8$, because the difference in the bar fraction between bulge-dominated galaxies and disk-dominated galaxies ( 25\%, Fig. 9) is much larger than the decline in the bar fraction with redshift (8\%, Table 3$)$. This is also illustrated in Fig. 19, where we plot the bar fraction as a function of Hubble type for a low and high redshift subsample. The figure shows that the bar fraction in bulge-dominated galaxies was already low at higher redshifts and implies that not many bars in these galaxies could have been dissolved since $z>0.60$. In addition, we observe that all morphological types contribute to the decline in the bar fraction by $8 \%$ from higher to lower redshifts (see Table 3).

Figure 19 seems to indicate that the $f_{\text {bar-morphology relation }}$ does not significantly change with redshift. This would suggest that bars are typically formed or destroyed during processes in which the morphology of the disk is emerging or changing. In other words, bars are not dissolved in, for instance, S0 galaxies, but can be destroyed during the processes in which a disk galaxy is transformed into a S0. On the other hand, a scenario in which bars are constantly formed and dissolved without altering the relative fractions across the Hubble sequence would also be consistent with our data. However, this would require a high degree of fine tuning between formation and destruction processes and is rather unlikely.

From a theoretical perspective, there are several studies indicating that present-day bars are rather robust and not easily destroyed (Shen \& Sellwood 2004; Athanassoula et al. 2005; Martinez-Valpuesta et al. 2006; Debattista et al. 2006).

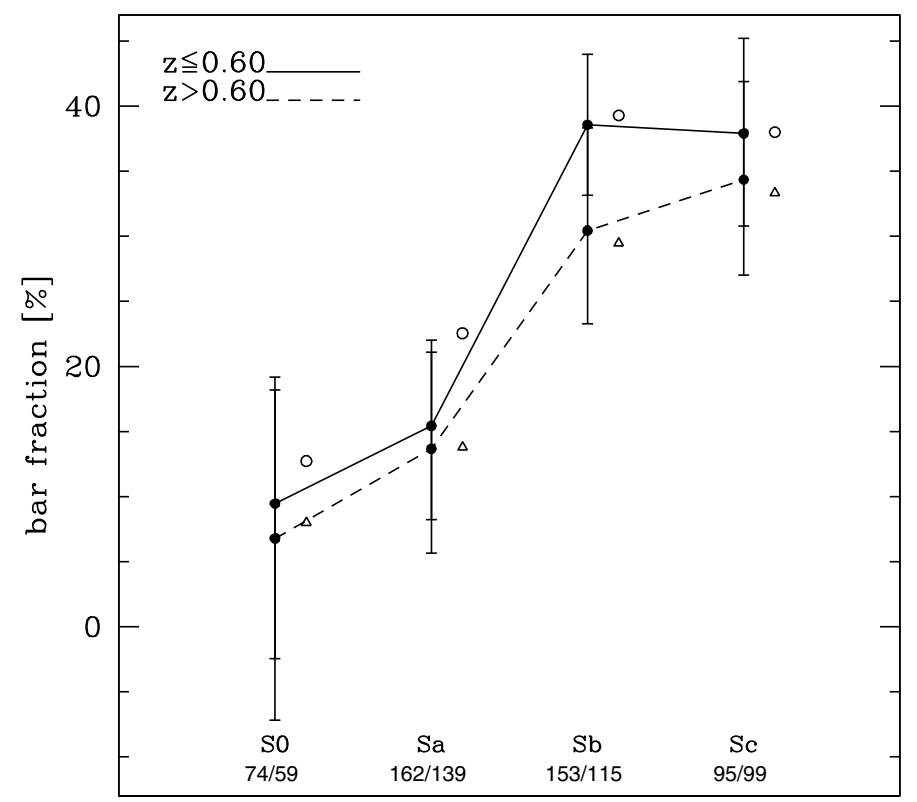

visual class

Fig. 19. The bar fraction as a function of Hubble type, separated into a subsample at $z \leq 0.60$ (solid line and open circles for the complete sample) and a subsample at $z>0.60$ (dashed line and open triangles for the complete sample). The numbers at the bottom of the plot indicate: number of galaxies at $z \leq 0.60 /$ number of galaxies at $z>0.60$. We omit Hubble types Sd and later, since the number of objects in these bins is too low. The error bars only include Poissonain errors.

Typically, a massive central mass concentration would be required to dissolve a bar. However, present-day super massive black holes, central dense stellar clusters, or the inner parts of bulges are not massive enough to affect bars significantly. However, it has been argued that a central mass concentration with the effects of gas inflows can lead to bar dissolution (Bournaud et al. 2005). In this picture, bars would become weaker and weaker and start to resemble lenses, which are preferentially found in early-type disks (Kormendy 1979; Kormendy \& Kennicutt 2004) and can be interpreted as dissolving bars. In this context, it is interesting to note that we find, on average, weaker bars in early-type disks compared to late-type disks (Fig. 17, lower panel). However, our results appear to be consistent with a scenario in which bars are rather stable and longlived, but the possibility that bar destruction and reformation also plays a crucial role cannot be ruled out.

\subsection{The distribution of barred galaxies in clusters}

In Sect. 4, we have shown that the bar fractions in clusters and in the field are essentially the same. This result indicates that clusters neither significantly foster nor prevent the formation of bars and if there are processes leading to the destruction of bars in clusters, they also act in the field. We point out that these results are based on relatively small samples and a confirmation with a larger sample would be desirable. Our findings are in agreement with the study by van den Bergh (2002), who investigated the bar fraction in field, group, and cluster environments. Considering the galaxy distribution in clusters, we have found that the bar fraction in the cluster center is higher than its average value (Fig. 18). Similar results were reported for local clusters (Thompson 1981; Andersen 1996). These findings indicate that 
the specific conditions in cluster centers support bar formation or help to avoid bar destruction. The large relative velocities of the galaxies in cluster centers prevent galaxy mergers, but galaxy flybys and the corresponding interactions are frequent. There is theoretical (Gerin et al. 1990; Mihos \& Hernquist 1994; Noguchi 1996) and observational (Elmegreen et al. 1990; Giuricin et al. 1993; Varela et al. 2004) evidence that galaxy-galaxy interactions trigger bar formation.

The triggered formation of bars in the cluster center could also be responsible for the size distribution of bars. Most galaxies hosting bars with sizes larger than the mean bar size for cluster galaxies $(3.68 \mathrm{kpc})$ are located within $R_{\mathrm{CC}} / R_{200}<0.5$ (Fig. 16). Although the number of objects studied is small, this is a remarkable result. Is it possible to relate the presence of these large bars at the cluster centers to the interactions proposed to instigate bar formation? One plausible scenario would be that the formation of these large bars was triggered in the cluster center and that the bars are therefore rather young. The fact that most of the host galaxies of these bars are of intermediate Hubble type appears to support this picture. Simulations of disk galaxies indicated that bars grow rapidly just after their formation (Berentzen et al. 2006) and become significantly shorter once the buckling instability occurs (Martinez-Valpuesta \& Shlosman 2004). Conversely, large bar sizes can also be interpreted as signs of maturity (Debattista \& Sellwood 2000; Valenzuela \& Klypin 2003) or just as an intermediate stage during the evolution of a barred disk galaxy (Curir et al. 2006). However, it is important to point out that the disk galaxies simulated in theoretical studies of bar formation and evolution are typically isolated or part of a cosmological simulation and the specific processes in clusters are not considered.

\section{Summary and conclusions}

We have searched for bars in 945 galaxies, drawn from a parent sample of 1906 disk galaxies from the EDisCS project. We used HST/ACS images taken in the $F 814 \mathrm{~W}$ filter and restricted our sample to $I_{\text {auto }}<23 \mathrm{mag}$. The selection of disk galaxies (S0-Sm) was based on their visual classifications. We identified and characterized bars based on ellipse fits to the surface brightness distribution and quantitative criteria. After excluding unsatisfactory fits and highly inclined systems $\left(>60^{\circ}\right)$, our bar analysis was performed for the remaining 945 disk galaxies. Spectroscopic observations were available for a subsample of 238 galaxies. Based on the corresponding redshifts and cluster assignments, we evaluated the distribution of barred disks in 11 galaxy clusters and 4 groups in the redshift range $0.4<z \leq$ 0.8 and analyzed the properties of their bars. Our main results were:

1. The total optical bar fraction, averaged over the entire sample covering the redshift range $z=0.4-0.8$, is $25 \%$ (20\% for strong bars, i.e., bar ellipticity $>0.4$ ). This is in good agreement with earlier studies at intermediate redshifts. The corresponding bar fractions for the spectroscopically based cluster and field samples are $24 \%$ and $29 \%$, respectively. Hence, the occurrence of bars in clusters is roughly the same as in the field.

2. We find that the bar fraction increases towards later Hubble types (form $\sim 10 \%$ for $\mathrm{S} 0$ to $>30 \%$ for $\mathrm{Sc}$ ). Interpreting this relation as being due to the decreasing prominence of the bulge, our result is in close agreement with the findings of Barazza et al. (2008). It suggests that the size (or mass) of the bulge has an impact on the probability that a bar forms or survives in a disk galaxy. The relation does not change with redshift, indicating that bars form or dissolve only when the disk changes its morphology.

3. The bar fraction as a function of effective radius exhibits a striking increase (from $\sim 15 \%$ to $\sim 45 \%$ ) towards larger radii. This result is expected in view of the morphology-bar fraction relation (point 2), but is much more pronounced than the latter. This indicates that it is really the structure of the disk that strongly affects bar formation and survival.

4. The bar size and bar ellipticity distributions of our sample galaxies are similar to those of other studies. The majority of bars have sizes $<5 \mathrm{kpc}$, as expected for un-deprojected samples. The average bar size is rather constant along the Hubble sequence ( $a_{\text {bar }}=2.5-3.5 \mathrm{kpc}$ ), while the average bar ellipticity increases towards later Hubble types (from $e_{\mathrm{bar}}<$ 0.4 for $\mathrm{S} 0$ to $e_{\text {bar }}>0.6$ for $\mathrm{Sd} / \mathrm{Sm} / \mathrm{Im}$ ). We suspect that this result is strongly affected by the fact that large and luminous bulges cause the ellipticities in the bar region to be lower.

5. We find a somewhat higher bar fraction $(\sim 31 \%)$ close to the centers of the clusters than at larger clustercentric distances $(\sim 18 \%)$. This is consistent with earlier results for the Virgo and Coma clusters. Moreover, bars in clusters are on average longer than in the field and preferentially located close to the cluster center. Most bars with sizes above the average $\left(a_{\text {bar }}=3.68 \mathrm{kpc}\right)$ are located at $R_{\mathrm{CC}} / R_{200}<0.5$. We have to point out that these results are based on the relatively small sample of spectroscopically confirmed cluster members and might therefore be affected by small number statistics.

It is interesting that we have not found a significant difference in either bar fractions or properties between cluster and field galaxies, but that the distributions of barred galaxies and bar sizes inside clusters appears to be a function of clustercentric distance. The disk galaxy properties related to bars do not differ fundamentally between cluster and field environments, but the specific conditions in cluster centers appear to be favorable for bar formation and survival, and even for the creation of relatively long bars.

Acknowledgements. This paper is based on observations collected at the European Southern Observatory, Chile, as part of large program 166.A-0162 (the ESO Distant Cluster Survey). The Dark Cosmology Centre is funded by the Danish National Research Foundation.

\section{References}

Abraham, R. G., Merrifield, M. R., Ellis, R. S., Tanvir, N. R., \& Brinchmann, J. 1999, MNRAS, 308, 569

Aguerri, J. A. L., \& González-García, A. C. 2009, A\&A, 494, 891

Aguerri, J. A. L., Mendez-Abreu, J., \& Corsini, E. M. 2009, A\&A, 495, 491

Andersen, V. 1996, AJ, 111, 1805

Athanassoula, E. 1992, MNRAS, 259, 328

Athanassoula, E. 2003, MNRAS, 341, 1179

Athanassoula, E., Lambert, J. C., \& Dehnen, W. 2005, MNRAS, 363, 496

Barazza, F. D., Jogee, S., \& Marinova, I. 2008, ApJ, 675, 1194

Berentzen, I., Shlosman, I., \& Jogee, S. 2006, ApJ, 637, 582

Bertin, E., \& Arnouts, S. 1996, A\&AS, 117, 393

Block, D. L., Bournaud, F., Combes, F., Puerari, I., \& Buta, R. 2002, A\&A, 394, L35

Bournaud, F., \& Combes, F. 2002, A\&A, 392, 83

Bournaud, F., Combes, F., \& Semelin, B. 2005, MNRAS, 364, L18

Bureau, M., \& Freeman, K. C. 1999, AJ, 118, 126

Buta, R., Vasylyev, S., Salo, H., \& Laurikainen, E. 2005, AJ, 130, 506

Buta, R., Laurikainen, E., Salo, H., Block, D. L., \& Knapen, J. H. 2006, AJ, 132, 1859

Combes, F., Debbasch, F., Friedli, D., \& Pfenniger, D. 1990, A\&A, 233, 82

Curir, A., Mazzei, P., \& Murante, G. 2006, A\&A, 447, 453

Debattista, V. P., \& Sellwood, J. A. 2000, ApJ, 543, 704

Debattista, V. P., Mayer, L., Carollo, C. M., et al. 2006, ApJ, 645, 209 
Desai, V., Dalcanton, J. J., Aragón-Salamanca, A, et al. 2007, ApJ, 660, 1151

de Vaucouleurs, G., de Vaucouleurs, A., Corwin, H. G., Jr., et al. 1991, Third Reference Catalogue of Bright Galaxies (Berlin, Heidelberg, New York: Springer-Verlag), Vol. 1-3, XII, 2069

Elmegreen, D. M., Elmegreen, B. G., \& Bellin, A. D. 1990, ApJ, 364, 415

Elmegreen, B. G., Elmegreen, D. M., Chromey, F. R., Hasselbacher, D. A., \& Bissell, B. A. 1996, AJ, 111, 2233

Elmegreen, B. G., Elmegreen, D. M., \& Hirst, A. C. 2004, ApJ, 612, 191

Erwin, P. 2005, MNRAS, 364, 283

Erwin, P., \& Sparke, L. S. 2002, AJ, 124, 65

Eskridge, P. B., Frogel, J. A., Pogge, R. W., et al. 2000, AJ, 119, 536

Eskridge, P. B., Frogel, J. A., Pogge, R. W., et al. 2002, ApJS, 143, 73

Finn, R. A., Zaritsky, D., McCarthy, D. W., Jr., et al. 2005, ApJ, 630, 206

Friedli, D., Wozniak, H., Rieke, M., Martinet, L., \& Bratschi, P. 1996, A\&AS, 118,461

Gadotti, D. A. 2008, MNRAS, 384, 420

Gerin, M., Combes, F., \& Athanassoula, E. 1990, A\&A, 230, 37

Giuricin, G., Mardirossian, F., Mezzetti, M., \& Monaco, P. 1993, ApJ, 407, 22

Halliday, C., Milvang-Jensen, B., Poirier, S., et al. 2004, A\&A, 427, 397

Hernández-Toledo, H. M., Vázquez-Mata, J. A., Martínez-Vázquez, L. A., et al. 2008, AJ, 136, 2115

Hunt, L. K., \& Malkan, M. A. 1999, ApJ, 516, 660

Jedrzejewski, R. I. 1987, MNRAS, 226, 747

Jogee, S., Kenney, J. D. P., \& Smith, B. J. 1999, ApJ, 526, 665

Jogee, S., Knapen, J. H., Laine, S., et al. 2002a, ApJ, 570, L55

Jogee, S., Shlosman, I., Laine, S., et al. 2002b, ApJ, 575, 156

Jogee, S., Barazza, F. D., Rix, H.-W., et al. 2004, ApJ, 615, L105

Jogee, S., Scoville, N., \& Kenney, J. D. P. 2005, ApJ, 630, 837

Knapen, J. H. 1999, The Evolution of Galaxies on Cosmological Timescales, ASP Conf. Ser., 187, 72

Knapen, J. H., Shlosman, I., \& Peletier, R. F. 2000, ApJ, 529, 93

Kormendy, J. 1979, ApJ, 227, 714

Kormendy, J. 1993, Galactic Bulges, IAU Symp., 153, 209

Kormendy, J., \& Kennicutt, R. C., Jr. 2004, ARA\&A, 42, 603

Kuijken, K., \& Merrifield, M. R. 1995, ApJ, 443, L13

Kumai, Y., Taniguchi, Y., \& Ishii, H. 1986, MNRAS, 223, 139

Laine, S., Shlosman, I., Knapen, J. H., \& Peletier, R. F. 2002, ApJ, 567, 97

Laurikainen, E., Salo, H., \& Rautiainen, P. 2002, MNRAS, 331, 880

Laurikainen, E., Salo, H., \& Buta, R. 2004, ApJ, 607, 103

Laurikainen, E., Salo, H., Buta, R., et al. 2006, AJ, 132, 2634

Lynden-Bell, D. 1979, MNRAS, 187, 101

Marinova, I., \& Jogee, S. 2007, ApJ, 659, 1176

Marinova, I., et al. 2009, ApJ, submitted

Martin, P. 1995, AJ, 109, 2428

Martinez-Valpuesta, I., \& Shlosman, I. 2004, ApJ, 613, L29
Martinez-Valpuesta, I., Shlosman, I., \& Heller, C. 2006, ApJ, 637, 214

Menéndez-Delmestre, K., Sheth, K., Schinnerer, E., Jarrett, T. H., \& Scoville, N. Z. 2007, ApJ, 657, 790

Mihos, J. C., \& Hernquist, L. 1994, ApJ, 431, L9

Milvang-Jensen, B., Noll, S., Halliday, C., et al. 2008, A\&A, 482, 419

Noguchi, M. 1996, ApJ, 469, 605

Odewahn, S. C. 1996, Barred Galaxies, IAU Colloq., 157, ASP Conf. Ser., 91, 30

Pelló, et al. 2008, A\&A, submitted

Pfenniger, D., \& Norman, C. 1990, ApJ, 363, 391

Pfenniger, D., \& Friedli, D. 1991, A\&A, 252, 75

Reese, A. S., Williams, T. B., Sellwood, J. A., Barnes, E. I., \& Powell, B. A. 2007, AJ, 133, 2846

Regan, M. W., \& Elmegreen, D. M. 1997, AJ, 114, 965

Regan, M. W., \& Teuben, P. J. 2004, ApJ, 600, 595

Regan, M. W., Sheth, K., \& Vogel, S. N. 1999, ApJ, 526, 97

Rudnick, G., Franx, M., Rix, H.-W., et al. 2001, AJ, 122, 2205

Rudnick, G., Rix, H.-W., Franx, M., et al. 2003, ApJ, 599, 847

Sakamoto, K., Okumura, S. K., Ishizuki, S., \& Scoville, N. Z. 1999, ApJ, 525, 691

Schinnerer, E., Maciejewski, W., Scoville, N., \& Moustakas, L. A. 2002, ApJ, 575,826

Sellwood, J. A., \& Wilkinson, A. 1993, Reports of Progress in Physics, 56, 173

Shen, J., \& Sellwood, J. A. 2004, ApJ, 604, 614

Sheth, K., Regan, M. W., Vogel, S. N., \& Teuben, P. J. 2000, ApJ, 532, 221

Sheth, K., Vogel, S. N., Regan, M. W., et al. 2002, AJ, 124, 2581

Sheth, K., Regan, M. W., Scoville, N. Z., \& Strubbe, L. E. 2003, ApJ, 592, L13

Sheth, K., Vogel, S. N., Regan, M. W., Thornley, M. D., \& Teuben, P. J. 2005, ApJ, 632, 217

Sheth, K., Elmegreen, D. M., Elmegreen, B. G., et al. 2008, ApJ, 675, 1141

Simard, L., Willmer, C. N. A., Vogt, N. P., et al. 2002, ApJS, 142, 1

Simard, L., et al. 2009, A\&A, submitted

Smail, I., Dressler, A., Couch, W. J., et al. 1997, ApJS, 110, 213

Thompson, L. A. 1981, ApJ, 244, L43

Valenzuela, O., \& Klypin, A. 2003, MNRAS, 345, 406

van den Bergh, S. 2002, AJ, 124, 782

van den Bergh, S. 2007, AJ, 134, 1508

van den Bergh, S., Cohen, J. G., Hogg, D. W., \& Blandford, R. 2000, AJ, 120, 2190

Varela, J., Moles, M., Márquez, I., et al. 2004, A\&A, 420, 873

Weinberg, M. D. 1985, MNRAS, 213, 451

White, S. D. M., Clowe, D. I., Simard, L., et al. 2005, A\&A, 444, 365

Whyte, L. F., Abraham, R. G., Merrifield, M. R., et al. 2002, MNRAS, 336, 1281

Zheng, X. Z., Hammer, F., Flores, H., Assémat, F., \& Rawat, A. 2005, A\&A, 435,507 